\title{
Modelling single cell electroporation with bipolar pulse pa- rameters and dynamic pore radii
}

\author{
Sadhana Talele*1, Paul Gaynor ${ }^{2}$, Michael J. Cree ${ }^{1}$, Jethro van Ekeran ${ }^{1}$ \\ ${ }^{1}$ Department of Electronic Engineering, University of Waikato, Private Bag 3105, Hamilton, New Zealand \\ ${ }^{2}$ Department of Electrical and Computer Engineering, University of Canterbury, Private Bag 4800, Christchurch, New \\ Zealand \\ Email: Sadhana Talele*-sadhana@waikato.ac.nz; \\ ${ }^{*}$ Corresponding author
}

\begin{abstract}
We develop a model of single spherical cell electroporation and simulate spatial and temporal aspects of the transmembrane potential and pore radii as an effect of any form of applied electric field. The extent of electroporation in response to sinusoidal electric pulses of two different frequencies in a range of extracellular conductivity for two different cell radii are compared. Results show that pore radii tend to be more normalized for $A C$ fields. The relative difference in fractional pore area is reduced by the use of a $1 \mathrm{MHz}$ sinusoidal applied electric field over a $100 \mathrm{kHz}$ field.
\end{abstract}

\section{Introduction}

Exposure of biological cells to electric fields can lead to a variety of biophysical and biochemical responses [1-7]. Electroporation (EP-often also referred to as electropermeabilization) is a phenomenon in which applied electric field pulses create transient nanometre-scale pores in a cell membrane, when the transmembrane potential $V m$ exceeds a semi-critical value creating a state of high permeability [8,9]. These pores limit further growth of $\mathrm{Vm}$. The pores are long lived, sometimes surviving in the membrane 
for up to several minutes, and providing pathways for the movement of large ions and high molecular weight molecules such as drugs and DNA into the cell $[8,9]$. These properties have resulted in electroporation as a common tool in biotechnology and is used in many applications of biotechnology, biochemistry, molecular biology, medicine and other biological research [7-18]. Some of the applications are as follows:

Electrochemotherapy (ECT): In cancer chemotherapy, some of the drugs do not show their anti-tumour effects because of insufficient transport through the cell membrane [7]. A combined use of chemotherapeutic drugs and application of electric pulse is known as electrochemotherapy and is useful for local tumour control. Especially, bleomycin has been reported to have shown a 700-fold increased cytotoxicity when used in ECT $[19,20]$. This helps to achieve a substantial anti-tumour effect with a small amount of the drug, which limits its side effects [21]. Bleomycin and cisplatin have proven to be much more effective in electrochemotherapy than in standard chemotherapy when applied to tumor cell lines in vitro, as well as in vivo on tumors in mice [22-24]. Clinical trials have been carried out with encouraging results $[11,13,25-28]$.

Electrogenetransfection (EGT): Application of electroporation for transfer of DNA into cells to effect some form of gene therapy, often referred to as electrogenetransfection, is currently being applied in some clinical trials. It is presently considered to have large potential as a non viral method to deliver genetic material into cells, the process aimed at correcting genetic diseases [29].

Electrofusion (EF): Under appropriate physical conditions, delivery of electric pulses can lead to membrane fusion in close-contact adjacent cells. EF results in the encapsulation of both original cells' intracellular material within a single enclosed membrane and can be used to produce genetic hybrids or hybridomas [30]. Hybridomas are hybrid cells produced by the fusion of an antibody secreting stimulated B-lymphocytes, with a tumour cell that grows well in culture. The hybridoma is then able to continue to grow in culture, and a large amount of specific desired antibodies can be recovered after processing. Electrofusion has proved to be a successful approach in the production of vaccines [31,32], antibodies [12], and reconstructed embryos in mammalian cloning [33]. Transdermal drug delivery (TDD): Application of high-voltage pulses to the skin allows a large increase in induced ionic and molecular transport across the skin barrier [34]. This has been applied for transdermal delivery of drugs, such as metoprolol [35], and also works for larger molecules, for example, DNA oligonucleotides [35].

Electroinsertion (EI): Another application of electroporation is insertion of molecules into the cell 
membrane. As the electric field induced membrane pores reseal, they entrap some of the transported molecules. Experiments on electroinsertion suggest the possibility of using the process to study certain physiological properties of these cells and understanding aspects of the lipid-protein interactions of the cell plasma membrane [36].

Electroporation is an important technique for transport of macromolecules such as genes, antibodies, and drugs, into a host cell. For effective transport, the pores should have sufficiently large radii, remain open long enough, and should not cause membrane rupture. In applications such as electrofusion and in-vivo electroporation, it is often necessary that cells of different radii be electroporated. Until recently, the development of theoretical models of electroporation lagged behind experimental research. In order to optimize the efficiency of electroporation, it is important to consider as many biological and physical aspects as possible and it is a necessity that a variety of electric field pulse parameters be tried. Thus, a comprehensive model which can predict electropermeabilization as a result of any form of applied electric field pulse and other important electroporation parameters is necessary. A number of models have been reported in literature. A model [37] is reported which analyses the time course of induced $V m$ when a cell is exposed to different time varying electric field shapes [37]. This model uses passive complex dielectric material based Laplace Transform equations to describe the transmembrane potential of the model cell. While this model is capable of producing results for arbitrary waveforms, it does not include the dynamic effects of membrane electropermeablization on $\mathrm{Vm}$ and pore density, $N$. Another theoretical model has been developed for single cell electroporation when exposed to a unipolar square shape electric field pulse [9]. This model considers electropermeabilization as a dynamic process and describes the evolution of $\mathrm{Vm}$ and $\mathrm{N}$, assuming a set of defined electroporation parameters. While this model includes the non-linear effects of membrane breakdown, it is not able to accommodate arbitrary pulse waveforms.

Another recent and substantially novel model based on nodal circuit analysis techniques includes both basic non-linear effects of electroporation and can provide results for arbitrary pulse waveforms in both single cell and tissue-like systems [38]. This model was primarily created to investigate the general response of cells to electric fields, so it does not specifically concentrate on the finer temporal or spatial components of electroporation.

Recent reported studies $[9,39]$ model EP to calculate transmembrane potential $\left(V_{m}\right)$ and the pore density $(N)$. These models consider the non-linear behaviour of EP, which is due to the dynamics of membrane pore formation and its effect on the transmembrane potential in turn. However, these models assume a 
single non-varying pore radius around the cell membrane. Other models [40,41] include spatial and temporal aspects of pore radius evolution. The electric fields used in these models were limited to unipolar DC pulses. However, oscillating pulse protocols have been reported to be used in electroporation based applications due to its advantage over DC pulse protocol [42] especially for electroporating cells with nonhomogeneous sizes. Thus the need to develop a model which can consider AC fields.

The study presented here develops a model of single spherical cell electroporation that simulates spatial and temporal aspects of pore radius as an effect of any given form of applied electric field (including unipolar or bipolar), and other important electroporation system parameters.

In particular, we first model a single spherical cell of $15 \mu$ m radius, under the application of a $2 \mu$ s DC unipolar pulse and two-cycles of a $1 \mathrm{MHz}$ sinusoidal AC (bipolar) pulse. The transmembrane potential and pore radius at various polar angular positions about the cell membrane and as a function of time are presented.

We then consider two cell radii $(15 \mu \mathrm{m}$ and $7.5 \mu \mathrm{m})$ and calculate fractional pore area (FPA) to compare the extent of elctropermabilization at varying extracellular conductivities and the effect of higher frequency sinusoidal AC pulses.

The simulation results are used to compare the extent of electroporation in response to sinusoidal AC electric field pulses of two different frequencies in a range of extracellular conductivity for the two cell radii. The results show that the pore radii tend to be more normalized (less widely spread) when an AC field is used when compared to a DC field. It is also observed that a $1 \mathrm{MHz}$ bipolar sinusoidal applied electric field pulse reduces the relative difference in fractional pore area between the two cell sizes compared to a $100 \mathrm{kHz}$ pulse. However, a significantly higher amplitude is required to create the same level of average fractional pore area.

\section{Model of a single cell}

Consider a spherical cell of radius $a$ with intracellular conductivity $\sigma_{\text {in }}$. The cell is immersed in a medium with conductivity $\sigma_{\mathrm{ex}}$. This system is exposed to a time-varying electric field $E(t)$. Azimuthal symmetry about the axis of the applied electric field is assumed. 


\subsection{Transmembrane potential}

It is assumed that the intracellular and extracellular regions are charge free. The homogeneous external electric field of strength $E(t)$ is used as a boundary condition, the potential being fixed at

$$
\Phi(3 a, \theta)=-3 a E(t) \cos \theta
$$

on a sphere of radius $3 a$ surrounding the cell, where $\theta$ is the polar angle. Since there are no free charges inside the region, the potential obeys Laplace's equation

$$
\nabla^{2} \Phi=0
$$

except at the cell membrane at a radial distance of $r=a$, where the potential is discontinuous because of the abrupt change in conductivity and the approximated infinitely thin membrane. Thus internal and external potentials can be defined as,

$$
\Phi(r, \theta)= \begin{cases}\Phi_{\text {in }}(r, \theta) & r<a, \\ \Phi_{\mathrm{ex}}(r, \theta) & a<r<3 a .\end{cases}
$$

The current across the membrane is used to relate the internal and external potentials [9], that is,

$$
-\hat{\mathbf{r}} \cdot\left(\sigma_{\mathrm{in}} \nabla \Phi_{\mathrm{in}}(a, \theta)\right)=-\hat{\mathbf{r}} \cdot\left(\sigma_{\mathrm{ex}} \nabla \Phi_{\mathrm{ex}}(a, \theta)\right)=C_{m} \frac{\partial V_{m}}{\partial t}+J_{m}
$$

where

$$
V_{m}(\theta)=\Phi_{\text {in }}(a, \theta)-\Phi_{\mathrm{ex}}(a, \theta) .
$$

Here $\hat{\mathbf{r}}$ is the unit outward radial vector, $C_{m}$ is the specific membrane capacitance, $V_{m}$ is the transmembrane potential and $J_{m}$ is the current density at the cell membrane due to existing pores. This current density is made up of three terms as follows,

$$
J_{m}=J_{\text {ion }}+J_{\text {sml }}+J_{\text {lge }}
$$

where $J_{\text {ion }}=g_{m}\left(V_{m}-V_{\text {rest }}\right)$ is the ionic current density [9] $\left(g_{m}\right.$ is the specific membrane conductance, and $V_{\text {rest }}$ is the membrane rest potential), $J_{\mathrm{sml}}$ is the current density through all the small (smaller than $1 \mathrm{~nm}$ radius) pores and $J_{\text {lge }}$ is the total current density through all the larger pores. These two later terms are explained with relevant equations as follows:

Current density through small pores $\left(J_{\mathrm{sml}}\right)$ : The model assumes that initially small pores are formed and the current density through these small pores $J_{\text {sml }}$ and is given [9] by

$$
J_{\mathrm{sml}}=N i_{\mathrm{sml}}(r),
$$


where $N$ is the pore density of the initial small pores formed and $i_{\mathrm{sml}}(r)$ is the diffusion current through a single pore of radius $r$ (true for small pores less than $1 \mathrm{~nm}$ only). A previously derived expression for $i_{\mathrm{sml}}[9]$, based upon the Nernst-Planck equation, is used for pore radius below $1 \mathrm{~nm}$, and is,

$$
i_{\mathrm{sml}}=\frac{\pi r^{2} \sigma_{\mathrm{ps}} v_{m} R T}{F h} \cdot \frac{\left(e^{v_{m}}-1\right)}{\left(G_{-} e^{v_{m}}-G_{+}\right)}
$$

with

$$
G_{ \pm}=\frac{w_{0} e^{w_{0} \pm n v_{m}} \pm n v_{m}}{w_{0} \pm n v_{m}}
$$

Here $\sigma_{\mathrm{ps}}$ is the conductivity of the aqueous solution that fills the pore (approximated by $\sqrt{\sigma_{\text {in }} \sigma_{\mathrm{ex}}}$ ), $F$ is Faraday's constant, $R$ is the universal gas constant, $T$ is the absolute temperature, $h$ is the thickness of the membrane, $w_{0}$ is the energy barrier inside the pore, $n$ is the relative entrance length of the pore and $v_{m}$ is the nondimensional transmembrane potential [9] given by

$$
v_{m}=V_{m}\left(\frac{F}{R T}\right) .
$$

Thus Equation 8 for pore current $i_{\text {sml }}$ accounts for the electrical interactions between the ions and the pore wall [9].

Current density through larger pores $\left(J_{\text {lge }}\right)$ : Initially formed small pores evolve in size if appropriate conditions exist. Now, assume $Q$ larger pores exist, and $i_{\text {lge }}$ is the current through individual electropores larger than $1 \mathrm{~nm}$. Then, $J_{\text {lge }}$ is the total current density through $Q$ larger pores; $r_{q}$ being the radius of the $q^{\text {th }}$ pore. Hence,

$$
J_{\text {lge }}=\frac{1}{A} \sum_{q=1}^{Q} i_{\text {lge }}\left(r_{q}\right)
$$

where $A$ is the corresponding cell surface area. For these larger pores, the current-voltage relationship assumes that the transmembrane potential $V_{m}$ occurs across the sum of pore resistance $R_{p}$ and the series input resistance $R_{\text {in }}[40,43]$, as follows:

$$
i_{\text {lge }}(r)=\frac{V_{m}}{R_{p}+R_{\text {in }}}
$$

where

$$
R_{p}=\frac{h}{\pi \sigma_{\mathrm{ps}} r_{q}^{2}}
$$

and

$$
R_{\mathrm{in}}=\frac{1}{2 \sigma_{\mathrm{ps}} r_{q}}
$$




\subsection{Formation of pores:}

Initially pores are assumed to be formed with the minimum-energy radius $r_{m}=0.76 \mathrm{~nm}$, given by [9]

$$
\frac{d N}{d t}=\psi e^{\left(V_{m} / V_{\mathrm{ep}}\right)^{2}}\left(1-\frac{N}{N_{\mathrm{eq}}\left(V_{m}\right)}\right)
$$

where $N$ is the pore density of the initial small pores formed, $\psi$ is the creation rate coefficient and $N_{\text {eq }}$ is the equilibrium pore density for a voltage $V_{m}$ given by

$$
N_{\mathrm{eq}}\left(V_{m}\right)=N_{0} e^{b\left(V_{m} / V_{\mathrm{ep}}\right)^{2}} .
$$

Here, $N_{0}$ is the initial pore density with no applied electric field, $V_{\mathrm{ep}}$ is the characteristic voltage of electroporation and $b$ is the pore creation constant [44].

\subsection{Evolution of pore radii:}

The pores that are initially created with minimum-energy radius $r_{m}$ change in size. This change supports minimizing the energy of the entire lipid bilayer [45]. Evolution of the radius of a pore is governed by equations 17-21 [40]. The lipid bilayer energy depends on a number of parameters involving the pore radius, electrostatic interaction between the lipid heads, pore edge energy, membrane tension and the electric force acting on the pore. The lipid bilayer energy $w_{m}$ is given by

$$
w_{m}=\sum_{q=1}^{Q}\left[w_{\mathrm{st}}\left(\frac{r_{*}}{r_{q}}\right)^{4}+2 \pi w_{\mathrm{ed}} r_{q}-\pi \xi_{\mathrm{eff}}\left(A_{p}\right) r_{q}^{2}+\int_{0}^{r_{q}} F\left(r, V_{m}\right) d r\right]
$$

The terms in this equation are explained in the following paragraphs.

The lipid head groups which line the pore interior, tend to repel each other due to steric and/or electrostatic interactions [45-47] and are taken into account by the first term in equation 17, where $w_{\mathrm{st}}$ is the steric repulsion energy [44].

The appearance of a circular pore in a membrane is balanced by the presence of two competing energy terms: reduction in energy barrier proportional to removal of pore area $\pi r_{q}^{2}$ (third term in equation 17) and increase in energy barrier by a linear edge component proportional to pore edge of length $2 \pi r_{q}$ [48-50] (second term in equation 17). Here, $w_{\text {ed }}$ is the pore edge energy, and $\xi_{\text {eff }}$ the effective tension of the membrane is given by

$$
\xi_{\text {eff }}\left(A_{p}\right)=2 \xi^{\prime}-\frac{2 \xi^{\prime}-\xi_{0}}{\left(1-\frac{A_{p}}{A}\right)^{2}}
$$


where $\xi^{\prime}$ is the energy per area of the hydrocarbon-water interface $[44,46], \xi_{0}$ is the tension of a membrane without pores and $A$ is the total area of the lipid bilayer. A varying value of the total area $A_{p}$ occupied by the pores at any given time is given by

$$
A_{p}=\sum_{q=1}^{Q} \pi r_{q}^{2}
$$

and contributes to a dynamic effect of pore formation both temporally and spatially.

The last term in equation 17 is the contribution of the membrane potential to the bilayer energy [44]. Assuming the inner surface of a pore as toroidal [44,51], the electric force $F$ acting on the pore is given by

$$
F\left(r, V_{m}\right)=\frac{F_{\max }}{\left(1+\frac{r_{h}}{r+r_{t}}\right)} V_{m}^{2} .
$$

This equation is a heuristic approximation [44] of the numerical solution which the authors have computed for the electrical force acting on a pore derived from first principles; here, $r_{h}$ and $r_{t}$ are constants taken from reference [44]. This equation is thought to be appropriate for larger pores as it predicts that $F$ approaches a constant value $F_{\max }$ as the pore radius increases, rather than increase linearly [50], or decrease to zero [52-54] as radius increases.

The rate of change of pore radii is given by [40]

$$
\frac{d r_{q}}{d t}=-\frac{D}{k T} \frac{\partial w_{m}}{\partial r_{q}}, \quad q=1,2, \ldots, Q
$$

where $D$ is the diffusion coefficient of the pore radius, $k$ is Boltzman's constant and $T$ is the absolute temperature. All parameter values are as given in Table 1.

\subsection{Limitations of the model}

The model described in this chapter is capable of simulating transmembrane potential and pore radii formation/evolution when a cell is exposed to any form of electric field, but only for spherical cells. The model does not include media permittivity. Including other shapes of cells and the media permittivity would require substantial changes to the model. The effect of other physical and chemical properties of electropermeabilization system are not simulated. Although the electrical parameters have the greatest effect on electroporation dynamics, the other properties may need to be included at some stage in an ultimate model of electroporation. Such inclusion of additional parameters may provide additional information. 


\section{Numerical Implementation}

The model described above is implemented in MATLAB. The following description of model geometry is illustrated in Figure 1. Azimuthal symmetry about the axis of the applied electric field is assumed. Extracellular space of thickness $2 a$ is assumed. The intra and extracellular space is discretized using spherical co-ordinates, such that $r_{i}=i \Delta r(i=1, \ldots, 51)$ and $\theta_{j}=j \Delta \theta(j=0, \ldots, 24)$, thus $\Delta r=3 a / 51$ and $\Delta \theta=\pi / 24$. Due to azimuthal symmetry, discretization in $\theta$ results in 25 circular rings on the surface of the cell. Each circular ring is modelled as a single variable. Initially, each circular ring of the cell membrane is assigned a pore density $N_{0}$, of 'small pores', each of the pores having identical radius $r_{m}$, the minimum energy radius. Each ring also has a transmembrane voltage $V_{m}$ calculated as given below.

The evolution of pore radii is calculated as follows. $N_{j}$ is the pore density for the $j^{\text {th }}$ ring on the cell membrane. In accordance with Equations 17 to 21, the pore radius evolves. An array of radii variables, one element per large pore, keeps track of these larger pore radii. The algorithm computes $\frac{d r_{q}}{d t}$ for the $q^{\text {th }}$ pore at every time-step. If this quantity is positive, small pores expand in size.

If $\frac{d r_{q}}{d t}>0$, then for the $j^{\text {th }}$ ring with area $A_{j}$, an integer number of large pore variables, namely floor $\left(N_{j} A_{j}\right)$ are created. The small pore population is decreased by the corresponding number of large pores that are created. To ensure that each pore is of a distinct value, the large pores are created at random radii normally distributed about the minimum energy radius $r_{m}$, with an extremely small standard deviation (order of $10^{-20} \mathrm{~m}$ ). An explicit Euler method is used for solving the first order differential equations.

The time step needs to be much smaller than the reciprocal of the highest frequency used for simulation. All the results presented here use a time step of $2 \mathrm{~ns}$, which is small enough to provide an accuracy that does not noticeably improve with smaller time steps [55]. Thus error bars are not included in any of the results.

For the calculation of $V_{m}$, the potential field $\Phi(r, \theta, t)$ is modelled in and around the cell as follows to calculate $\Phi_{\text {in }}$ and $\Phi_{\text {ex }}$. The finite difference method is used to solve Laplace's equation in a sphere surrounding the cell to give the electric potential. When discretized on $r$ and $\theta$ as described above, Laplace's equation becomes a large set of linear (algebraic) equations relating the values of $\Phi$ at the discrete points. The external electric field is used as a boundary condition at $r=3 a$. Current across the membrane is used as a boundary condition at the cell membrane.

Laplace's equation written in spherical coordinates with the assumption of azimuthal symmetry can be 
reduced to,

$$
\frac{\partial^{2} \Phi}{\partial r^{2}}+\frac{2}{r} \frac{\partial \Phi}{\partial r}+\frac{1}{r^{2}} \frac{\partial^{2} \Phi}{\partial \theta^{2}}+\frac{\cot \theta}{r^{2}} \frac{\partial \Phi}{\partial \theta}=0
$$

For points on the boundary of this region, at $r=3 a, \Phi$ is made equal to the value set by the boundary condition. For interior points not on the membrane, the derivatives are approximated by finite differences of the cell, which gives the system of linear equations,

$$
\begin{aligned}
\frac{\partial \Phi\left(r_{i}, \theta_{j}\right)}{\partial r} & \approx \frac{\Phi\left(r_{i+1}, \theta_{j}\right)-\Phi\left(r_{i-1}, \theta_{j}\right)}{2 \Delta r} \\
\frac{\partial^{2} \Phi\left(r_{i}, \theta_{j}\right)}{\partial r^{2}} & \approx \frac{\Phi\left(r_{i+1}, \theta_{j}\right)-2 \Phi\left(r_{i}, \theta_{j}\right)+\Phi\left(r_{i-1}, \theta_{j}\right)}{\Delta r^{2}} \\
\frac{\partial \Phi\left(r_{i}, \theta_{j}\right)}{\partial \theta} & \approx \frac{\Phi\left(r_{i}, \theta_{j+1}\right)-\Phi\left(r_{i}, \theta_{j-1}\right)}{2 \Delta \theta} \\
\frac{\partial^{2} \Phi\left(r_{i}, \theta_{j}\right)}{\partial \theta^{2}} & \approx \frac{\Phi\left(r_{i}, \theta_{j+1}\right)-2 \Phi\left(r_{i}, \theta_{j}\right)+\Phi\left(r_{i}, \theta_{j-1}\right)}{\Delta \theta^{2}} .
\end{aligned}
$$

Substituting the discrete derivatives above into Laplace's equation (equation 2) and simplifying gives,

$$
\begin{aligned}
-2\left[\Delta r^{2}+r_{i}^{2} \Delta \theta^{2}\right] \Phi\left(r_{i}, \theta_{j}\right)+r_{i} \Delta \theta^{2}\left[r_{i}-\Delta r\right] \Phi\left(r_{i-1}, \theta_{j}\right) & \\
+ & r_{i} \Delta \theta^{2}\left[r_{i}+\Delta r\right] \Phi\left(r_{i+1}, \theta_{j}\right)+\Delta r^{2}\left[1-\frac{1}{2} \cot \left(\theta_{j}\right) \Delta \theta\right] \Phi\left(r_{i}, \theta_{j-1}\right) \\
& +\Delta r^{2}\left[1+\frac{1}{2} \cot \left(\theta_{j}\right) \Delta \theta\right] \Phi\left(r_{i}, \theta_{j+1}\right)=0 .
\end{aligned}
$$

In order to include the boundary condition involving the current at the cell membrane, the radial derivatives of $\Phi_{\text {in }}$ and $\Phi_{\text {ex }}$ must be estimated. Assume $\alpha$ is the index such that $r_{\alpha}=a$ (that is, the radial index that gives the point on the cell membrane, see figure 1). Consider $\frac{\partial}{\partial r} \Phi_{\text {in }}\left(r, \theta_{j}\right)$ which is approximated at $r=a-\frac{1}{2} \Delta r$ and $r=a-\frac{3}{2} \Delta r$ by

$$
\begin{aligned}
&\left.\frac{\partial \Phi_{\mathrm{in}}}{\partial r}\right|_{\left(a-\frac{\Delta r}{2}, \theta_{j}\right)} \approx \frac{\Phi_{\mathrm{in}}\left(r_{\alpha}, \theta_{j}\right)-\Phi_{\mathrm{in}}\left(r_{\alpha-1}, \theta_{j}\right)}{\Delta r} \\
& \text { and }\left.\quad \frac{\partial \Phi_{\mathrm{in}}}{\partial r}\right|_{\left(a-\frac{3 \Delta r}{2}, \theta_{j}\right)} \approx \frac{\Phi_{\mathrm{in}}\left(r_{\alpha-1}, \theta_{j}\right)-\Phi_{\mathrm{in}}\left(r_{\alpha-2}, \theta_{j}\right)}{\Delta r}
\end{aligned}
$$

respectively. We can estimate $\frac{\partial}{\partial r} \Phi_{\text {in }}\left(a, \theta_{j}\right)$ by linearly extrapolating from these two points out to the membrane itself, so that

$$
\begin{aligned}
\left.\frac{\partial \Phi_{\text {in }}}{\partial r}\right|_{\left(a, \theta_{j}\right)} & \left.\approx \frac{\partial \Phi_{\text {in }}}{\partial r}\right|_{\left(a-\frac{\Delta r}{2}, \theta_{j}\right)}+\frac{1}{2}\left(\left.\frac{\partial \Phi_{\text {in }}}{\partial r}\right|_{\left(a-\frac{\Delta r}{2}, \theta_{j}\right)}-\left.\frac{\partial \Phi_{\text {in }}}{\partial r}\right|_{\left(a-\frac{3 \Delta r}{2}, \theta_{j}\right)}\right) \\
& \approx \frac{3 \Phi_{\text {in }}\left(r_{\alpha}, \theta_{j}\right)-4 \Phi_{\text {in }}\left(r_{\alpha-1}, \theta_{j}\right)+\Phi_{\text {in }}\left(r_{\alpha-2}, \theta_{j}\right)}{2 \Delta r} .
\end{aligned}
$$


Similarly $\frac{\partial}{\partial r} \Phi_{\mathrm{ex}}(a, \theta)$ is computed at the membrane similarly to give,

$$
\left.\frac{\partial \Phi_{\mathrm{ex}}}{\partial r}\right|_{\left(a, \theta_{j}\right)} \approx \frac{-3 \Phi_{\mathrm{ex}}\left(r_{\alpha}, \theta_{j}\right)+4 \Phi_{\mathrm{ex}}\left(r_{\alpha+1}, \theta_{j}\right)-\Phi_{\mathrm{ex}}\left(r_{\alpha+2}, \theta_{j}\right)}{2 \Delta r} .
$$

All these finite difference equations along with the boundary conditions are expressed as a system of linear equations. They are solved at each time step, using the LU-decomposition method to give the solution of the Laplace equation. The value of $V_{m}$ is calculated as $V_{m}=\Phi_{\text {in }}-\Phi_{\text {ex }}$ at the membrane at each $\theta_{j}$. Depending on the parameter values a high number of pores may be formed. The total simulation time depends on the number of large pores created by the applied electric pulse. Strong pulses may create many pores, thus requiring long simulation times. To speed up computation in the event of such large numbers of pores forming (i.e. $>10^{4}$ ), pores are 'created in groups' of identical pore radius. A single radius variable describes the radius of all pores in a group, thus reducing the number of radius variables required. The size of this group is selected as required, based on the total number of pores and number of variables the computer can handle.

To confirm the accuracy and validate the model presented here, the model results for the transmembrane potential $V_{m}$ were initially compared with results obtained by calculating the transmembrane potential $V_{m}$ using analytical solutions given by [56]. Solutions to the numerical model and the analytical model were obtained for applied bipolar sinusoidal pulse electric fields $(20 \mathrm{kV} / \mathrm{m}$ and $40 \mathrm{kV} / \mathrm{m}$ at a frequency of $100 \mathrm{kHz})$ and $(40 \mathrm{kV} / \mathrm{m}, 60 \mathrm{kV} / \mathrm{m}$ and $80 \mathrm{kV} / \mathrm{m}$ at a frequency of $1 \mathrm{MHz})$, each amplitude is less than that required for onset of electroporation, as the analytical solution does not model the non-linear effects of membrane electroporation. This confirms that the developed numerical model is a valid numerical calculation technique. The model by [41] includes provision for non-linear electroporation dynamics including pore radius evolution to a unipolar electric field step. The induced transmembrane potential at $\theta=0$ is very close to that reported by [41], indicating that the pore dynamics had been correctly calculated in the model presented here. [41] has not presented the induced transmembrane potential evolution at other angular positions of the cell and thus cannot be compared.

Induced transmembrane potential and pore radii evolution to a bipolar electric field is not available in literature. The simulation results of this chapter present these.

\section{Simulation Results}

Details of temporal and spatial evolution of transmembrane potential and pore radius for bipolar pulses have not been reported in the literature to date. Thus, simulations are carried out for evolution of 
transmembrane potential and pore radius for two applied electric field pulses (DC and AC) for a single spherical cell. All the simulations are done with a time step of $2 \mathrm{~ns}$, thus resulting in a set of acurately repeatable set of values. Thus error bars have not been required to be included.

Of particular interest is the cell charging time, pore creation time and pore radius evolution time [41]. Cell charging is the time from first application of external electric field to the formation of the first additional pore on the cell membrane. Pore creation phase is time from the first pore to the time of the last pore formed. The remaining time in the simulation is the pore radius evolution time.

\subsection{Evolution of transmembrane potential, formation of pores, and pore radius evolution}

Figures 2 to 9 show the transmembrane potential and the pore radius evolution around the circumference of a $15 \mu \mathrm{m}$ radius cell in two individual cases: when a $2 \mu \mathrm{s}, 94.7 \mathrm{kV} / \mathrm{m}$ DC electric field pulse is applied, and when a sinusoidal AC (1 MHz), $235 \mathrm{kV} / \mathrm{m}$ amplitude electric field pulse is applied for the same length of time. The peak amplitude $\left(E_{p}\right)$ of the electric field in each case was set to provide a terminal FPA at the end of $2 \mu$ s of approximately $0.015 \%$ (presented in [57] as being within good range of permeabilization). All the other parameters in the model are kept identical for both simulations.

Figures 2 and 3 (DC case), and 6 and 7 (AC case) show the evolution of the transmembrane potential at various positions around the cell. Figures 4 and 5 (DC case) and 8 and 9 (AC case) show the evolution of pores at various positions around the cell. Each black line represents evolution of the first few pores modelled individually since there are only few additional pores formed at the beginning. Each green(grey) line represents evolution of a group of pores (groups of 20 pores in these simulations) as many pores begin to be created. The number in the right hand corner of these figures indicate the number of pores eventually formed at the particular polar $(\theta)$ position. At the angular positions not shown in these figures $\left(\theta=60^{\circ}\right.$ to $120^{\circ}$ ), there are no new pores created.

In the DC case, the time duration of $2 \mu \mathrm{s}$ is chosen for the simulation so that the fine temporal features of the relationships between the pore formation, pore radii evolution and transmembrane potential evolution can be seen. As observed in figures 4 and 5 the first pore is formed at position $\theta_{24}$ (at $0.42 \mu \mathrm{s}$ ) and the last pore for this particular pulse protocol is formed at position $\theta_{6}$ (at $1.3 \mu \mathrm{s}$ ). This constitutes the pore creation phase $(0.42 \mu \mathrm{s}-1.3 \mu \mathrm{s})$. The initial period when no pores are formed $(0-0.42 \mu \mathrm{s})$ is the charging of the cell membrane. Once transmembrane potential reaches a magnitude of about $1 \mathrm{~V}$, pores start to form and expand/evolve. These time ranges for charging and pore creation are specific to parameters chosen in this simulation, and will vary with changes in the parameters. 
In the case when an AC field is applied, as seen in figures 8 and 9, additional pores may form at the subsequent peak of transmembrane potential. This happens if the transmembrane potential at the particular position is beyond the threshold value. This increases the pore creation time as compared with DC pulses.

\subsection{Frequency dependence of electropermeabilization in a heterogeneous population of cell radii/sizes.}

Owing to the variable nature of biological growth dynamics, all electroporation applications treat cells of a range of sizes. Although this range may only be in the order of a few percent, some applications may have ranges exceeding 200 percent [58]. As such, whatever the application, there may be a substantial benefit in being able to normalize the degree of electropermeabilization (considered equivalent to the fractional pore area) with respect to cell radius/size. Earlier passive and dynamic modelling of bipolar electric field induced transmembrane potential has indicated that as the frequency of a constant amplitude applied electric field is increased, transmembrane potential reduces and becomes less dependent on cell radius $[39,59]$. In order to achieve a transmembrane potential high enough for EP, the electric field amplitude must be increased at higher frequencies. To determine whether this effect translates to dynamic electroporation modelling that includes pore radii variability, two higher frequency electric field pulses of $100 \mathrm{kHz}$ and $1 \mathrm{MHz}$ were used for electropermeabilization simulation. Two substantially (yet realistically) different cell radii of $15 \mu \mathrm{m}$ and $7.5 \mu \mathrm{m}$ were considered.

Figures 10 and 11 show the fractional pore area for $7.5 \mu \mathrm{m}$ and $15 \mu \mathrm{m}$ radius cells exposed to a two-cycle sine wave electric field pulse of $100 \mathrm{kHz}$ and $1 \mathrm{MHz}$ respectively. The peak amplitude of the electric field, $E_{p}$ was set to provide an average terminal fractional pore area (for the two cell radii) of approximately $0.015 \%$ at a $\sigma_{\mathrm{ex}}$ of $0.2 \mathrm{~S} / \mathrm{m}$. It is seen from figures 10 and 11 that the relative difference in FPA between the two cell sizes at an extracellular conductivity of $0.2 \mathrm{~S} / \mathrm{m}$ is 1.1 for $100 \mathrm{kHz}$ and 0.6 for $1 \mathrm{MHz}$. This relative difference increases as $\sigma_{\mathrm{ex}}$ increases to a limit of approximately 1 . For a physiological medium with $\sigma_{\mathrm{ex}}=1.2 \mathrm{~S} / \mathrm{m}$ (as found in in vivo $\mathrm{EP}$ applications), this relative difference is 0.93 for the $1 \mathrm{MHz}$ two-cycle sine wave electric field pulse and 1.0 for the $100 \mathrm{kHz}$ electric field pulse when aiming at a mean fractional pore area of $0.015 \%$. 


\section{Discussion}

\subsection{Evolution of transmembrane potential and pore radius}

For a DC pulse protocol, it is seen that during the charging phase, transmembrane potential $V_{m}$ has a larger magnitude at hyperpolarized pole (position $\theta=180^{\circ}$ ) as compared to the hypopolarized pole (position $\theta=0^{\circ}$ ) due to the negative membrane rest potential of $-80 \mathrm{mV}$. As an effect of this, the poration on the hyperpolarized side occurs earlier than the hypopolarized side, in agreement with reported experimental results in the literature [60]. The model also predicts that the DC pulse creates more but smaller pores on the hyperpolarized hemisphere (13406 pores) as against fewer and larger pores on the hypopolarized hemisphere (8282 pores). Similar modelling predictions are reported by [41] in the literature. This prediction agrees with the experimental results reported by [61], in which smaller molecules entered through hyperpolarized end while larger molecules through the hypopolarized end.

The number of pores at a particular position of the cell not only depends on the transmembrane potential, but also on the condition of the remaining cell membrane. This is a plausible explanation as to why more pores are formed at $\theta=7.5^{\circ}$ and $\theta=15^{\circ}$ as well as at $\theta=172.5^{\circ}$ and $\theta=165^{\circ}$ as compared to position $\theta=0^{\circ}$ and $\theta=180^{\circ}$ respectively. The pores near to the non-polar regions tend to be larger in agreement with the modelling results presented by [41].

\subsection{Effect of field strength and frequency on pore dynamics}

An important aim of this study was to explore the effects of bipolar pulse protocols and their parameters on electroporation. In general the peak transmembrane potential on the cell membrane increases with increasing unipolar field strength up to a maximum value of around $1.43 \mathrm{~V}$. Transmembrane potential does not significantly exceed this limit because the net amount of electroporation (which is measured by the final fractional pore area) depends sensitively on transmembrane potential. The transmembrane potential increases initially by capacitive charging. New pores in the membrane form and expand/evolve due to electroporation and allow current to flow. This prevents further increase of the transmembrane potential. Fractional pore area is also found to increase with increasing electric field magnitude. This is because additional pores form until their total area allows sufficient current to flow to stop the increase of transmembrane potential. We also note that the radii of the largest pores are smaller for stronger applied fields. The fractional pore area increases in spite of this because, for strong fields, very many smaller pores (radii in the range $1-5 \mathrm{~nm}$ ) are formed.

Similar trends are seen with bipolar (AC) pulse protocols at a fixed frequency. However, to produce a given 
fractional pore area value, higher bipolar field strengths must be used compared with unipolar (DC) field strengths for the same overall pulse duration. More succinctly then, for a fixed electric field amplitude, fractional pore area decreases with increasing frequency, as does the total number of pores. This is to be expected because electroporation can only occur when the field strength exceeds a certain threshold. For bipolar (AC) fields at higher frequencies, the time intervals during which electroporation can occur are clearly shorter.

For unipolar electric field pulses, the pores formed in electroporation have widely distributed radii (around 1-8 nm), with significant contributions to the total fractional pore area from pores with radii in different size-ranges. This is not the case for bipolar fields. For example, at $1 \mathrm{MHz}$, nearly all of the total fractional pore area is contributed by pores with radii $1-3.4 \mathrm{~nm}$.

It is evident from figures 10 and 11 that, considering $0.015 \%$ fractional pore area as the desirable fractional pore area, two-cycles of $1 \mathrm{MHz}$ sinusoidal bipolar electric field reduces the relative difference in fractional pore area between the two cell sizes compared to two-cycles of a $100 \mathrm{kHz}$ bipolar electric field. However, a significantly higher amplitude is required to create the same level of average fractional pore area. Lower values of extracellular conductivity are warranted for better normalization of the degree of electroporation (fractional pore area), as higher values of extracellular conductivity increase the relative difference in fractional pore area for cells of different radii/size. Since typical in vivo applications have an extracellular conductivity equal to the physiological conductivity in the region of $1.2 \mathrm{~S} / \mathrm{m} \mathrm{[37],} \mathrm{it} \mathrm{is} \mathrm{unlikely} \mathrm{that}$ normalization of the degree of electroporation can be achieved in these applications through utilization of higher frequency AC pulses. Typically, at higher extracellular conductivity, the required magnitude of electric field also reduces.

\section{Conclusion}

The model is capable of simulating transmembrane potential and pore radii formation/evolution when a cell is exposed to any form of electric field. The results show that the pore radii tend to be more normalized when a bipolar sinusoidal AC field pulse is used as compared to a DC field pulse of the same duration. As a result, larger pore radii formation is more likely when a DC unipolar field pulse is used. The results also show that better normalization of the degree of electroporation, measured as fractional pore area FPA, especially at lower values of extracellular conductivity, can be achieved with use of higher frequency (1 $\mathrm{MHz}$ ) bipolar electric field as compared to $100 \mathrm{kHz}$. Thus, choice of the electric field parameters (magnitude and frequency) and the extracellular conductivity for optimum electropermeabilization is a 
trade off between parameters like the desired pore radii values, and normalization of fractional pore area between the required cell radii/size.

The results of modelling study in this paper, especially use of ac electric field remain to be experimentally investigated. However, while this paper was through reviewing process, a recent publication [62] demonstrates experimental results of electroporation using ac electric fields. Their study confirms that efficient electroporation may be achieved using ac fields. This could be very well related to the simulation result presented in section 4.2. Analysis and measurements need high resolution both temporally and spatially. Use of pulsed laser fluorescence microscope with a sub microsecond imaging capability has been used by [60,63], although for DC electric fields. This method would be useful to observe transmembrane potential formation with ac pulses as simulated in this paper. Pore size information can be inferred from selective molecular transport across electroporated cell membrane as done by [61] for dc pulses. Through use of fluorescence measurement using flow cytometry [62], level of molecular uptake by electroporated cell as well as \% of cells electroporated in a heterogeneous mixture of cell radii, can be measured. These measurements could be related to the quantity FPA simulated in the paper presented here. Modern techniques like In vivo imaging of irreversible electroporation by means of electrical impedance tomography [64]are also coming up which will be helpful in imaging the simulation results reported here mre accurately.

\section{References}

1. Pavlin P, Pavŝelj N, Miklavĉiĉ D: Dependence of Induced Transmembrane Potential on cell Density, Arrangement, and Cell Position inside a Cell System. IEEE Transactions on Biomedical Engineering 2002, 49:605-612.

2. Neumann E: The relaxation hysteresis of membrane electroporation. In Electroporation and Electrofusion in Cell Biology. Edited by Neumann E, Sowers AE, Jordan CA, New York: Plenum Press 1989:61-82.

3. Mir LM: Therapeutic perspectives of in vivo cell electropermeabilization. Bioelectrochemistry 2000, 53:1-10.

4. Neumann E, Kakorin S, Toensing K: Membrane electroporation and electro-mechanical deformation of vesicles and cells. Faraday Discuss 1998, :111-125.

5. Neumann E, Kakorin S: Digression on membrane electroporation for drug and gene delivery. Technology in Cancer Research and Treatment 2002, 1:329-339.

6. Griese T, Kakorin S, Neumann E: Conductometric and electrooptic relaxation spectrometry of lipid vesicle electroporation. Physical Chemistry Chemical Physics 2002, 4:1217-1227.

7. Miklavciiê D, Kotnik T: Electroporation for electrochemotherapy and gene therapy. In Bioelectromagnetic Medicine. Edited by Markov MS, New York: Marcel Dekker 2004:637-656.

8. Dev SB, Widera DP, Hofmann GA: Medical applications of electroporation. IEEE Transactions on Plasma Science 2000, 28:206-223. 
9. DeBruin KA, Krassowska W: Modeling electroporation in a single cell. I. Effects of field strength and rest potential. Biophysical Journal 1999, 77:1213-1224.

10. Kotnik T, Pucihar G, Rebersek M, Mir LM, Miklavêiĉ D: Role of pulse shape in cell membrane electropermeabilization. Biochim Biophys Acta 2003, 1614:193-200.

11. Snoj M, Rudolf Z, Cemaẑar M, Jancar B, Serŝa G: Successful sphincter-saving treatment of anorectal malignant melanoma with electrochemotherapy, local excision and adjuvant brachytherapy. Anti-Cancer Drugs 2005, 16:345-348.

12. Schmidt E, Leinfelder U, Gessner P, Zillikens D, Brocker EB, Zimmermann U: CD19+ B lymphocytes are the major source of human antibody-secreting hybridomas generated by electrofusion. Journal of Immunological Methods 2001, 255:93-102.

13. Tozon N, Kodre V, Serŝa G, Cemaẑar M: Effective Treatment of Perianal tumors in Dogs with Electrochemotherapy. Anticancer Research 2005, 25:839-946.

14. Marty M, Sersa G, Garbay JR, Gehl J, Collins CG, Snoj M, Billard V, Geertsen PF, Larkin JO, Miklavcic D, Pavlovic I, Paulin-Kosir SM, Cemazar M, Morsli N, Soden DM, Rudolf Z, Robert C, O'Sullivan GC, Mir LM: Electrochemotherapy - An easy, highly effective and safe treatment of cutaneous and subcutaneous metastases: Results of ESOPE (European Standard Operating Procedures of Electrochemotherapy study). European Journal of Cancer Supplements 2006, 4:3-13.

15. Mir LM, Gehl J, Sersa G, Collins CG, Garbay JR, Billard V, Geertsen PF, Rudolf Z, O’Sullivan GC, Marty M: Standard operating procedures of the electrochemotherapy: Instructions for the use of bleomycin or cisplatin administered either systemically or locally and electric pulses delivered by the CliniporatorTM by means of invasive or non-invasive electrodes. European Journal of Cancer Supplements 2006, 4:14-25.

16. Li S (Ed): Electroporation Protocols: Preclinical and Clinical Gene Medicine. Humana Press 2008.

17. Ŝatkauskas S, Bureau MF, Puc M, Mahfoudi A, Scherman D, Miklavĉiĉ D, Mir LM: Mechanisms of in vivo DNA electrotransfer: Respective contributions of cell electropermeabilization and DNA electrophoresis. Molecular Therapy 2002, 5:133-140.

18. Heller LC, Heller R: In Vivo Electroporation for Gene Therapy. Human Gene Therapy 2006, 17:890-897.

19. Cemaẑar M, Miklavĉiĉ D, Serŝa G: Intrinsic sensitivity of tumor cells to bleomycin as an indicator of tumor response to electrochemotherapy. Japanese Journal of Cancer Research 1998, 89:328-333.

20. Serŝa G: Electrochemotherapy. In Electrochemotherapy, Electrogenetherapy, and Transdermal Drug Delivery: Electrically Mediated Delivery of Molecules to Cells (Methods in Molecular medicine). Edited by Jaroszeski MJ, Heller R, Gilbert R, Totowa, New Jersey: Humana Press 2000:119-133.

21. Serŝa G, Novaković S, Miklavciiĉ D: Potentiation of bleomycin antitumor effectiveness by electrotherapy. Cancer Letters 1993, 69:81-84.

22. Mir LM, Orlowski S, Belehradek J, Paoletti C: Electrochemotherapy potentiation of antitumour effect of bleomycin by local electric pulses. European Journal of cancer 1991, 27:68-72.

23. Mir LM, Orlowski S, Belehradek JJ, Teissié J, Rols M, Serŝa G, Miklavĉiĉ D, Gilbert R, Heller R: Biomedical applications of electric pulses with special emphasis on antitumor electrochemotherapy. Bioelectrochemistry and Bioenergetics 1995, 38:203-207.

24. Serŝa G, Cemaẑar M, Miklavĉiĉ D: Antitumor effectiveness of electrochemotherapy with cisdiamminedichloroplatinum(II) in mice. Cancer Research 1995, 55:3450-3455.

25. Glass LF, Fenske NA, Jaroszeski M, Perrott R, Harvey DT, Reintgen DS, Heller R: Bleomycin-mediated electrochemotherapy of basal cell carcinoma. Journal of the American Academy of Dermatology 1996, 34:82-86. 
26. Serŝa G, Stabuĉ B, Cemaẑar M, Miklavĉiĉ D, Rudolf Z: Electrochemotherapy with cisplatin: the systemic antitumour effectiveness of cisplatin can be potentiated locally by the application of electric pulses in the treatment of malignant melanoma skin metastases. Melanoma Research 2000, 10:381-385.

27. Gothelf A, Mir LM, Gehl J: Electrochemotherapy: Results of cancer treatment using enhanced delivery of bleomycin by electroporation. Cancer Treatment Reviews 2003, :1-17.

28. Kranjc S, Cemaẑar M, Grosel A, Sentjurc M, Serŝa G: Radiosensitising effect of electrochemotherapy with bleomycin in LPB sarcoma cells and tumors in mice. BMC Cancer 2005.

29. Budak-Alpdogan T, Banerjee D, Bertino JR: Hematopoietic stem cell gene therapy with drug resistance genes: An update. Cancer Gene Therapy 2005, 12:849-863.

30. Zimmermann U: Electric field-mediated fusion and related electrical phenomena. Biochim Biophys Acta 1982, 694:227-277.

31. Scott-Taylor TH, Pettengell R, Clarke I, Stuhler G, Barthe MCL, Walden P, Dalgleish AG: Human tumour and dendritic cell hybrids generated by electrofusion: Potential for cancer vaccines. Biochim Biophys Acta 2000, 1500:265-267.

32. Orentas R, Schauer D, Bin Q, Johnson BD: Electrofusion of a weakly immunogenic neuroblastoma with dendritic cells produces a tumor vaccine. Cellular Immunology 2001, 213:4-13.

33. Gaynor P, Wells DN, Oback B: Couplet alignment and improved electrofusion by dielectrophoresis for a zona-free high-throughput cloned embryo production system. Medical and Biological Engineering and Computing 2005, 43:150-154.

34. Prausnitz MR, Bose VG, Langer R, Weaver JC: Electroporation of mammalian skin: A mechanism to enhance transdermal drug delivery. Proceedings of the National Academy of Sciences 1993, 90:10504-10508.

35. Vanbever R, Lecouturier N, Preat V: Transdermal delivery of metoprolol by electroporation. Pharmacological Research 1994, 11:1657-1662.

36. Mouneimne Y, Tosi PF, Barhoumi R, Nicolau C: Electroinsertion: An electrical method for protein implantation into cell membranes. In Guide to Electroporation and Electrofusion. Edited by Chang DC, Chassy BM, Saunders JA, Sowers AE, San Diego, CA: Academic Press 1992:327-346.

37. Kotnik T, Miklavêice D, Slivnik T: Time course of transmembrane voltage induced by time-varying electric fields a method for theoretical analysis and its application. Bioelectrochemistry and Bioenergetics 1998, 45:3-16.

38. Gowrishankar TR, Weaver JC: An approach to electrical modeling of single and multiple cells. Proceedings of the National Academy of Sciences 2003, 100:3203-3208.

39. Talele S, Gaynor P: Nonlinear time domain model of electropermeabilization: Response of a single cell to an arbitrary applied electric field. Journal of Electrostatics 2007, 65:775-784.

40. Smith KC, Neu JC, Krassowska W: Model of Creation and Evolution of Stable Electropores for DNA Delivery. Biophysical Journal 2004, 86:2813-2826.

41. Krassowska W, Filev PD: Modeling electroporation in a single cell. Biophysical Journal 2007, 92:404-417.

42. Chang DC, Hunt JR, Zheng Q, Gao PQ: Electroporation and electrofusion using pulsed radio-frequency electric field. In Guide to Electroporation and Electrofusion. Edited by Chang DC, Chassy BM, Saunders JA, Sowers AE, San Diego, CA: Academic Press 1992:303-326.

43. Newman J: Resistance for flow of current to a disk. Journal of The Electrochemical Society 1966, 113:501-502. 
44. Neu JC, Krassowska W: Modeling postshock evolution of large electropores. Physical Review E 2003, 67:1-12.

45. Weaver JC, Chizmadzhev Y: Theory of electroporation: a review. Bioelectrochemistry and Bioenergetics 1996, 41:135-160.

46. Israelachvili J: Intermolecular and Surface Forces. London, UK: Academic Press 1992.

47. Neu JC, Krassowska W: Asymptotic model of electroporation. Physical Review E 1999, 59:3471-3482.

48. Weaver JC, Powell KT: Theory of electroporation. In Electroporation and Electrofusion in Cell Biology. Edited by Neumann E, Sowers AE, Jordan CA, New York: Plenum Press 1989:111-126.

49. Glaser RW, Leikin SL, Chernomordik LV, Pastushenko VF, , Sokirko AI: Reversible electrical breakdown of lipid bilayers: Formation and evolution of pores. Biochim Biophys Acta 1988, 940:275287.

50. Abidor IG, Arakelyan VB, Chernomordik LV, Chizmadzhev YA, Pastushenko VF, Tarasevich MR: Electric breakdown of bilayer membranes: I. The main experimental facts and their qualitative discussion. Bioelectrochemistry and Bioenergetics 1979, 6:37-52.

51. Kanduŝer M, Fô̂naria M, Ŝentjurc M, Kralj-Igliĉ V, Hägerstrand H, Igliĉ A, Miklavĉiĉ D: Effect of surfactant polyoxyethylene glycol $\mathbf{C}_{12} \mathbf{E}_{8}$ on electroporation of cell line DC3F. Colloids and Surfaces A 2003, 214:205-217.

52. Barnett A, Weaver JC: Electroporation: A unified, quantitative theory of reversible electrical breakdown and mechanical rupture in artificial planar bilayer membranes. Bioelectrochemistry and Bioenergetics 1991, 25:163-182.

53. Joshi RP, Hu Q, Schoenbach KH, Hjalmarson HP: Improved energy model for membrane electroporation in biological cells subjected to electrical pulses. Physical Review E 2002, 65(4):041920-041928.

54. Pastushenko VF, Chizmadzhev YA: Stabilization of conducting pores in BLM by electric current. General Physiology and Biophysics 1982, 1:43-52.

55. Garcia AL: Numerical methods for Physics. New Jersey: Prentice Hall 1994.

56. Holzapfel C, Vienken J, Zimmermamm U: Rotaion of cells in an electric field. Journal of Membrane Biology 1982, 67:13-26.

57. Hibino M, Shigemori M, Itoh H, Nagayama K, Kinosita K Jr: Membrane conductance of an electroporated cell analyzed by submicrosecond imaging of transmembrane potential. Biophysical Journal 1991, 59:209-220.

58. Foster KR, Schwan HP: Dielectric properties of tissues. In Handbook of biological effects of electromagnetic fields, second edition. Edited by Polk C, Postow E, Boca Raton, Florida: CRC Press 1996:25-97.

59. Grosse C, Schwan HP: Cellular membrane potentials induced by alternating fields. Biophysical Journal 1992, 66:1632-1642.

60. Hibino M, Itoh $\mathrm{H}$, Kinosita KJ: Time courses of cell electroporation as revealed by submicrosecond imaging of transmembrane potential. Biophysical Journal 1993, 64:1789-1800.

61. Tekle E, Astumian RD, Chock PB: Selective and asymmetric molecular transport across electroporated cell membranes. Proceedings of the National Academy of Sciences 1994, 91:11512-11516.

62. Chen C, Evans JA, Robinson MP, Smye SW: Measurement of the efficiency of cell membrane electroporation using pulsed ac fields. Phys. Med. Biol. 2008, 53:4747-4757.

63. K Kinosita J, Ashikawa I, Saita N, Yoshimura H, HItoh, Nagayama K, Ikegami A: Electroporation of cell membrane visualized under a pulsed-laser fluorescence microscope. Biophysical Journal 1988, 53:1015-1019. 
64. Granot Y, Ivorra A, Maor E, Rubinsky B: In vivo imaging of irreversible electroporation by means of electrical impedance tomography. Phys. Med. Biol. 2009, 54:4927-4943.

65. Kotnik T, Miklavciiĉ D: Theoretical evaluation of the distributed power dissipation in biological cells exposed to electric fields. Bioelectromagnetics 2000, 21:385-394.

\section{Tables}

Table 1 - Geometric, electrical and electroporation parameters used in simulation for evolution of pore radii.

\begin{tabular}{|c|c|c|}
\hline \multicolumn{2}{|c|}{ Table 1 } \\
\hline$a$ & $15.0(\mu \mathrm{m})$ & cell radius \\
\hline$C_{m}$ & $10^{-2}\left(\mathrm{Fm}^{-2}\right)$ & specific membrane capacitance [41] \\
\hline$h$ & $5.0(\mathrm{~nm})$ & membrane thickness [9,37, 65] \\
\hline$g_{m}$ & $1.9\left(\mathrm{Sm}^{-2}\right)$ & specific membrane conductance [9] \\
\hline$V_{\text {rest }}$ & $-80(\mathrm{mV})$ & membrane rest potential [9] \\
\hline$\sigma_{\text {in }}$ & $0.3\left(\mathrm{~S} \mathrm{~m}^{-1}\right)$ & intracellular conductivity [37] \\
\hline$\sigma_{\text {ex }}$ & $1.2\left(\mathrm{Sm}^{-1}\right)$ & extracellular conductivity [65] \\
\hline$r_{*}$ & $0.51(\mathrm{~nm})$ & minimum radius of hydrophilic pores [40] \\
\hline$r_{m}$ & $0.76(\mathrm{~nm})$ & minimum energy radius at $V_{m}=0$ [40] \\
\hline$T$ & $295(\mathrm{~K})$ & absolute room temperature [9] \\
\hline$n$ & 0.15 & relative entrance length of pores [9] \\
\hline$b$ & 2.46 & pore creation constant [9] \\
\hline$V_{\mathrm{ep}}$ & $258(\mathrm{mV})$ & characteristic voltage of electroporation [9] \\
\hline$N_{0}$ & $1.5 \times 10^{9}\left(\mathrm{~m}^{-2}\right)$ & initial pore density [9] \\
\hline$w_{0}$ & 2.65 & energy barrier within pore [9] \\
\hline$\psi$ & $1 \times 10^{9}\left(\mathrm{~m}^{-2} \mathrm{~s}^{-1}\right)$ & creation rate coefficient [9] \\
\hline$w_{\mathrm{st}}$ & $1.4 \times 10^{-19}(\mathrm{~J})$ & steric repulsion energy [40] \\
\hline$w_{\text {ed }}$ & $1.8 \times 10^{-11}\left(\mathrm{~J} \mathrm{~m}^{-1}\right)$ & edge energy [40] \\
\hline$\xi_{0}$ & $1 \times 10^{-6}\left(\mathrm{~J} \mathrm{~m}^{-2}\right)$ & tension of the bilayer without pores [41] \\
\hline$\xi^{\prime}$ & $2 \times 10^{-2}\left(\mathrm{~J} \mathrm{~m}^{-2}\right)$ & tension of hydrocarbon-water interface [9] \\
\hline$F_{\text {max }}$ & $0.70 \times 10^{-9}\left(\mathrm{~N} \mathrm{~V}^{-2}\right)$ & max electric force for $V_{m}=1 \mathrm{~V}$ [9] \\
\hline$r_{h}$ & $0.97 \times 10^{-9}(\mathrm{~m})$ & constant [40] \\
\hline$r_{t}$ & $0.31 \times 10^{-9}(\mathrm{~m})$ & constant [40] \\
\hline$D$ & $5 \times 10^{-14}\left(\mathrm{~m}^{-2} \mathrm{~s}^{-1}\right)$ & diffusion coefficient for pore radius [41] \\
\hline
\end{tabular}




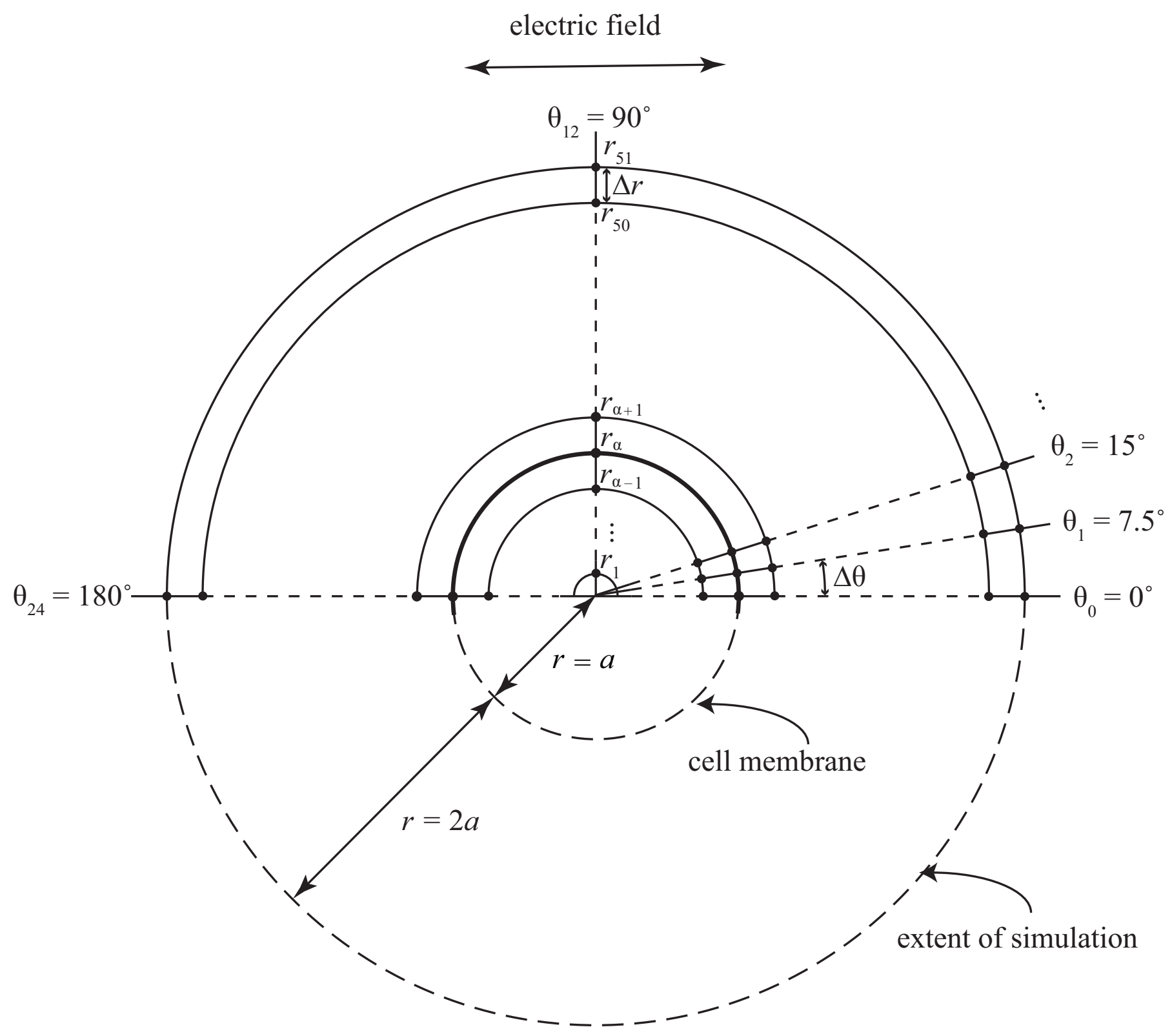

Figure 1: Spherical cell model geometry showing the radius and angle discretization used in the numerical model 


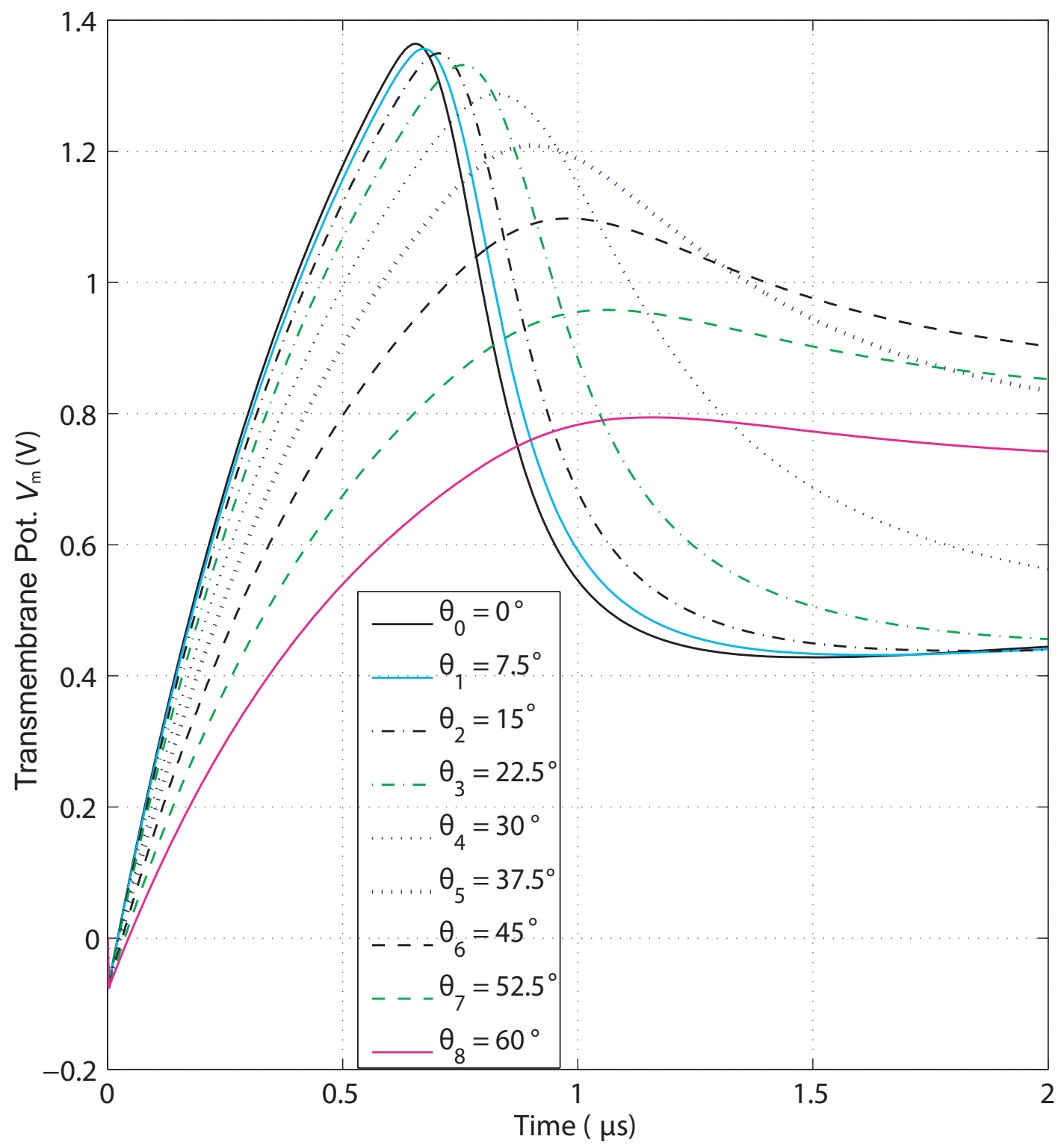

Figure 2: Transmembrane potential evolution at angles $0^{\circ}$ to $60^{\circ}$ around the cell membrane for a DC applied electric field pulse of $94.7 \mathrm{kV} / \mathrm{m}$ magnitude. 


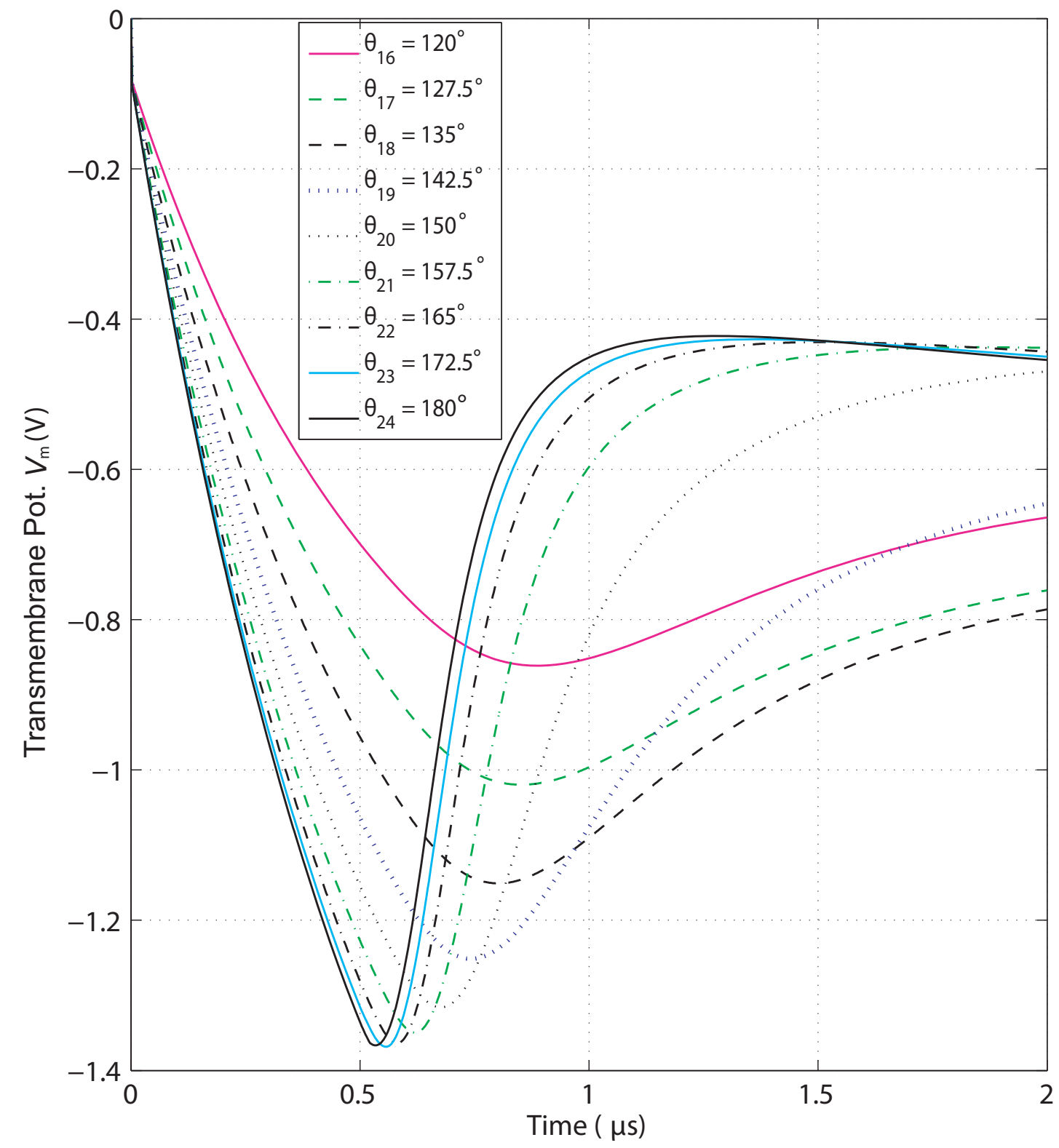

Figure 3: Transmembrane potential evolution at angles $120^{\circ}$ to $180^{\circ}$ around the cell membrane for a DC applied electric field pulse of $94.7 \mathrm{kV} / \mathrm{m}$ magnitude. 
$\theta_{0}=0^{\circ}$

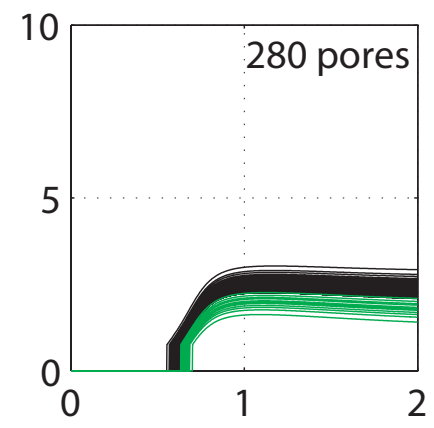

$\theta_{3}=22.5^{\circ}$

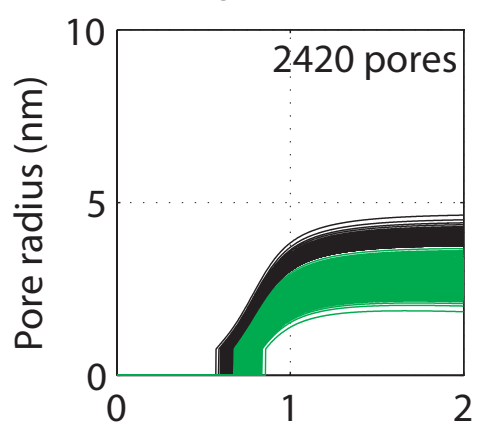

$\theta_{6}=45^{\circ}$

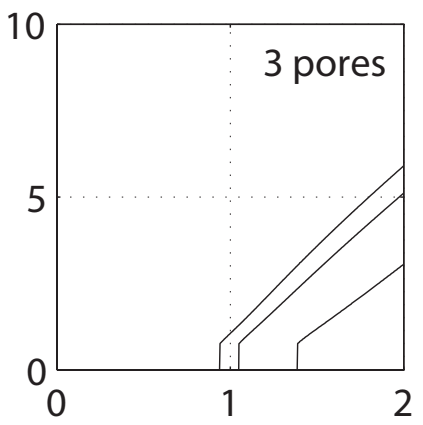

$\theta_{1}=7.5^{\circ}$

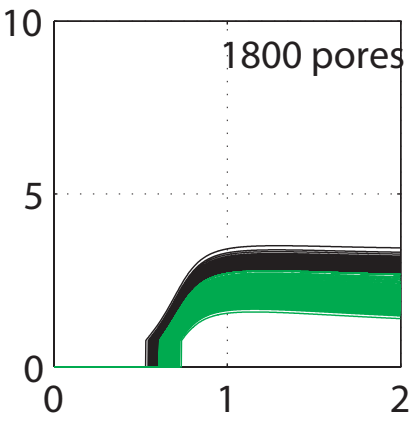

$\theta_{4}=30^{\circ}$

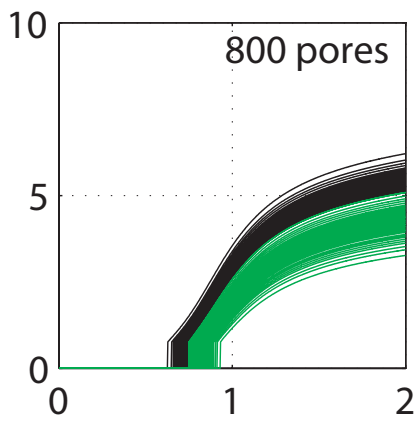

$\theta_{7}=52.5^{\circ}$

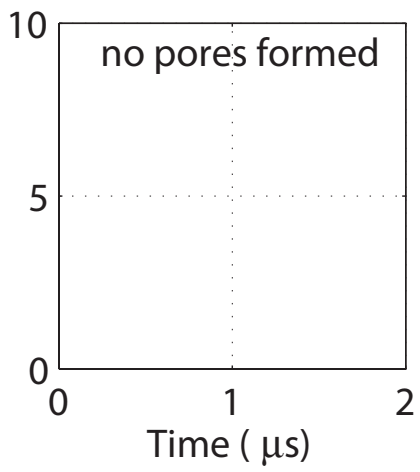

$\theta_{2}=15^{\circ}$

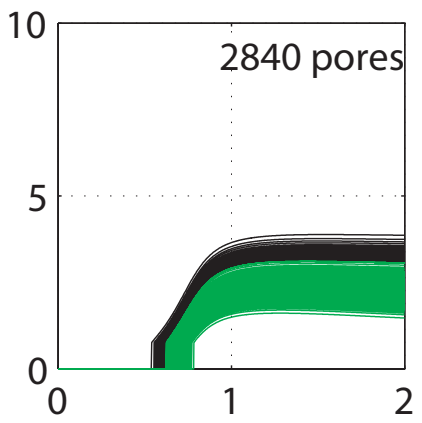

$\theta_{5}=37.5^{\circ}$

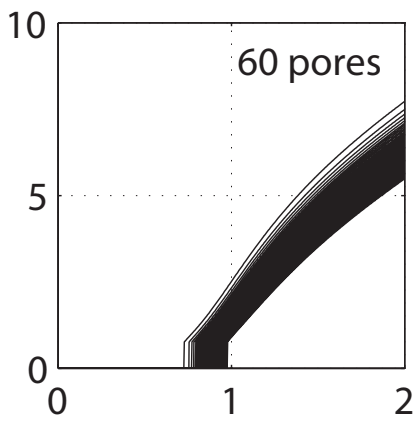

$\theta_{8}=60^{\circ}$

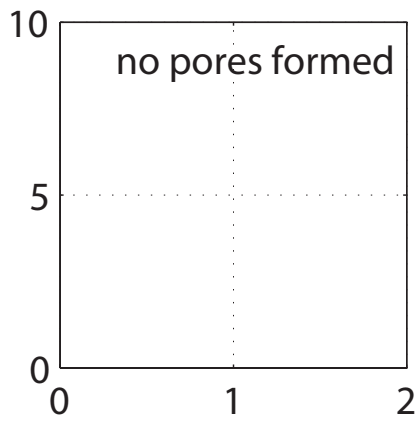

Figure 4: Pore radius evolution at angles $0^{\circ}$ to $60^{\circ}$ around the cell membrane for a DC applied electric field pulse of $94.7 \mathrm{kV} / \mathrm{m}$ magnitude. Black lines represent individual pores, green(grey) lines represent groups of 20 pores. 
$\theta_{16}=120^{\circ}$

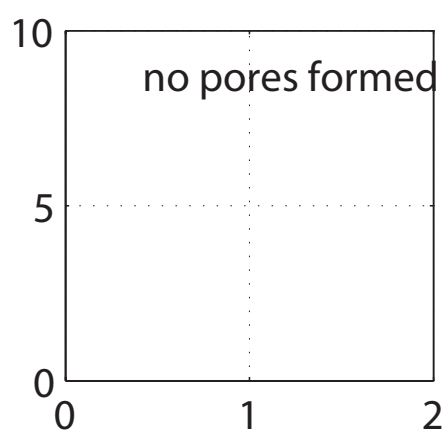

$\theta_{19}=142.5^{\circ}$

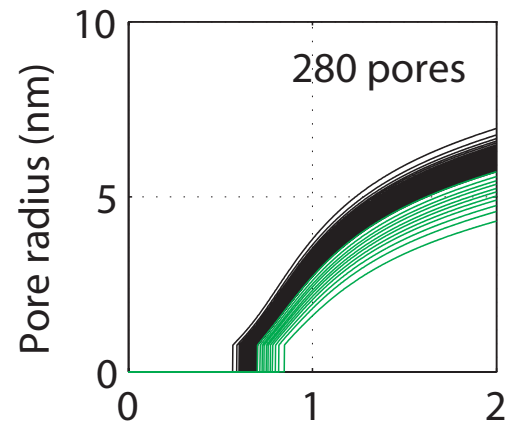

$\theta_{22}=165^{\circ}$

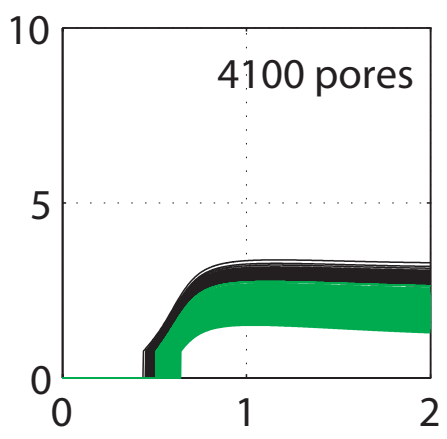

$\theta_{17}=127.5^{\circ}$

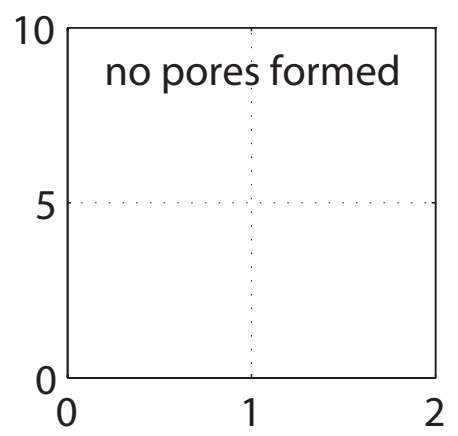

$\theta_{20}=150^{\circ}$

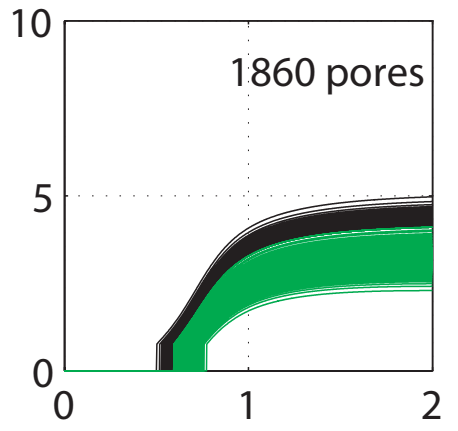

$\theta_{23}=172.5^{\circ}$

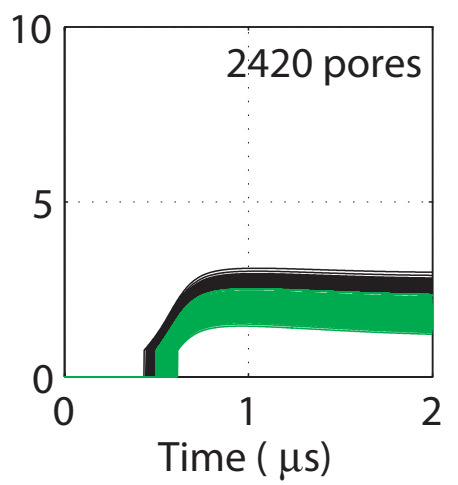

$\theta_{18}=135^{\circ}$

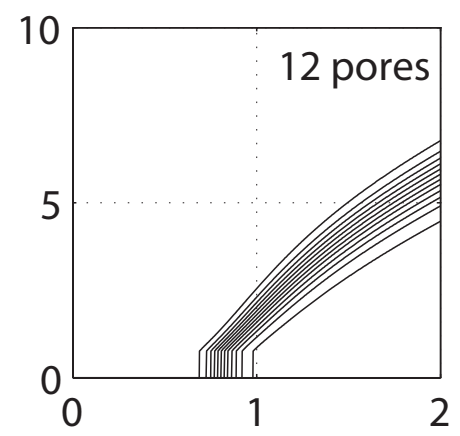

$\theta_{21}=157.5^{\circ}$

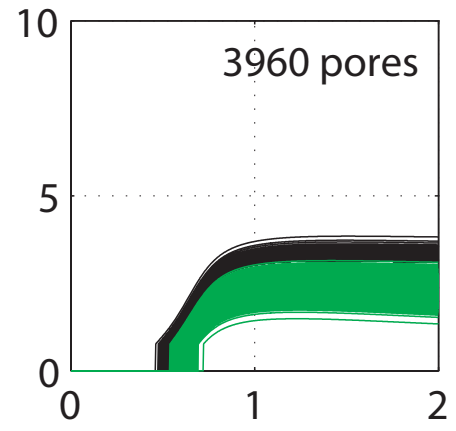

$\theta_{24}=180^{\circ}$

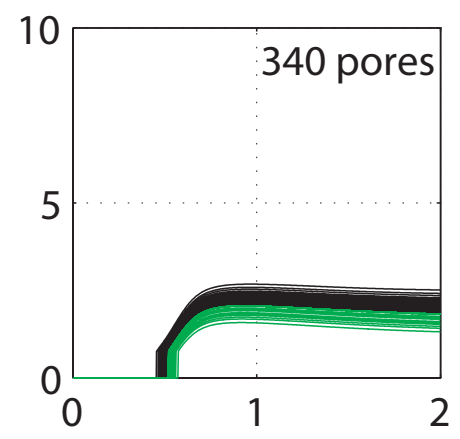

Figure 5: Pore radius evolution at angles $120^{\circ}$ to $180^{\circ}$ around the cell membrane for a DC applied electric field pulse of $94.7 \mathrm{kV} / \mathrm{m}$ magnitude. Black lines represent individual pores, green(grey) lines represent groups of 20 pores. 


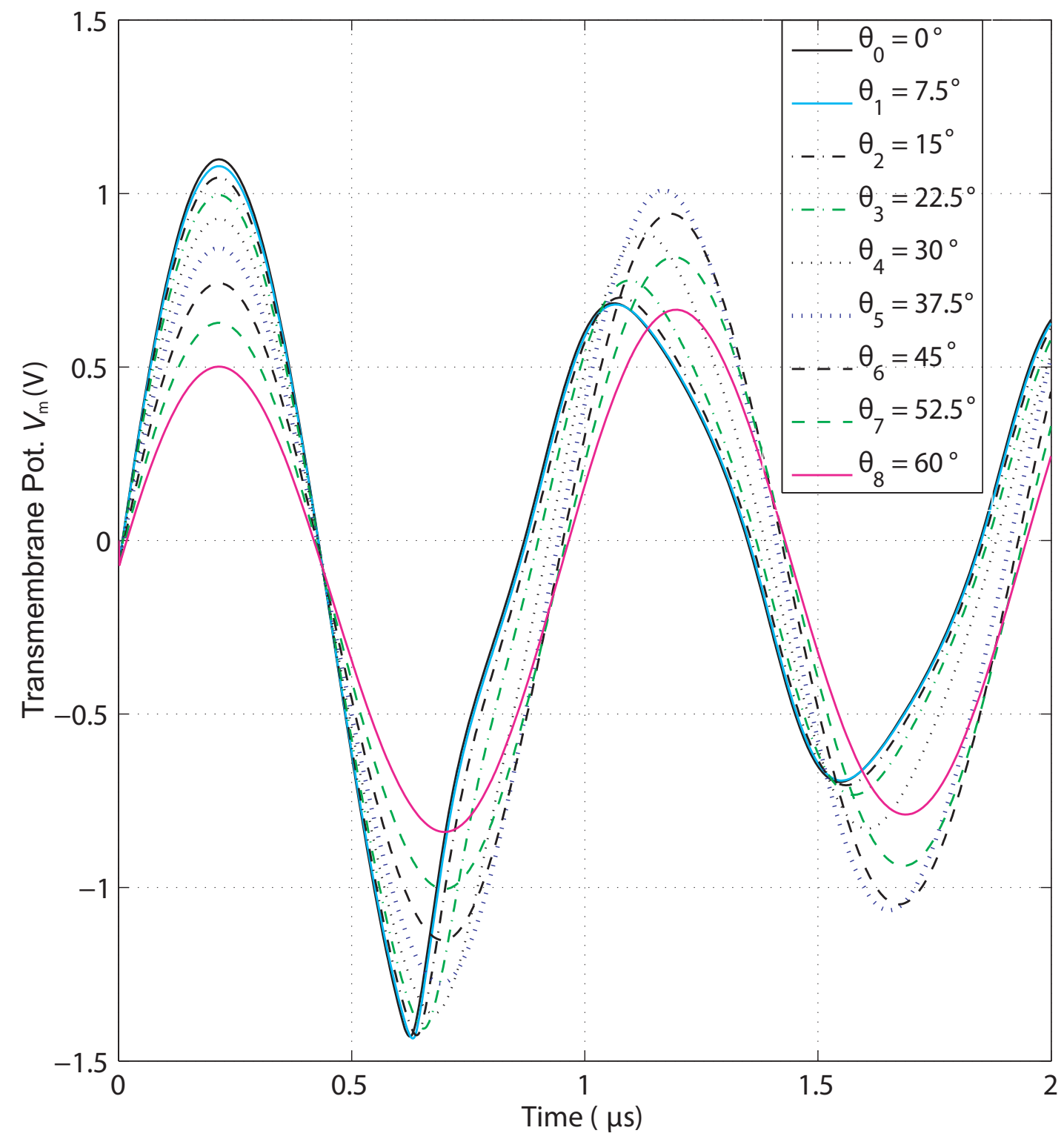

Figure 6: Transmembrane potential evolution at angles $0^{\circ}$ to $60^{\circ}$ around the cell membrane for a two-cycle $1 \mathrm{MHz}$ sinusoidal bipolar applied electric field pulse with $235 \mathrm{kV} / \mathrm{m}$ peak amplitude. 


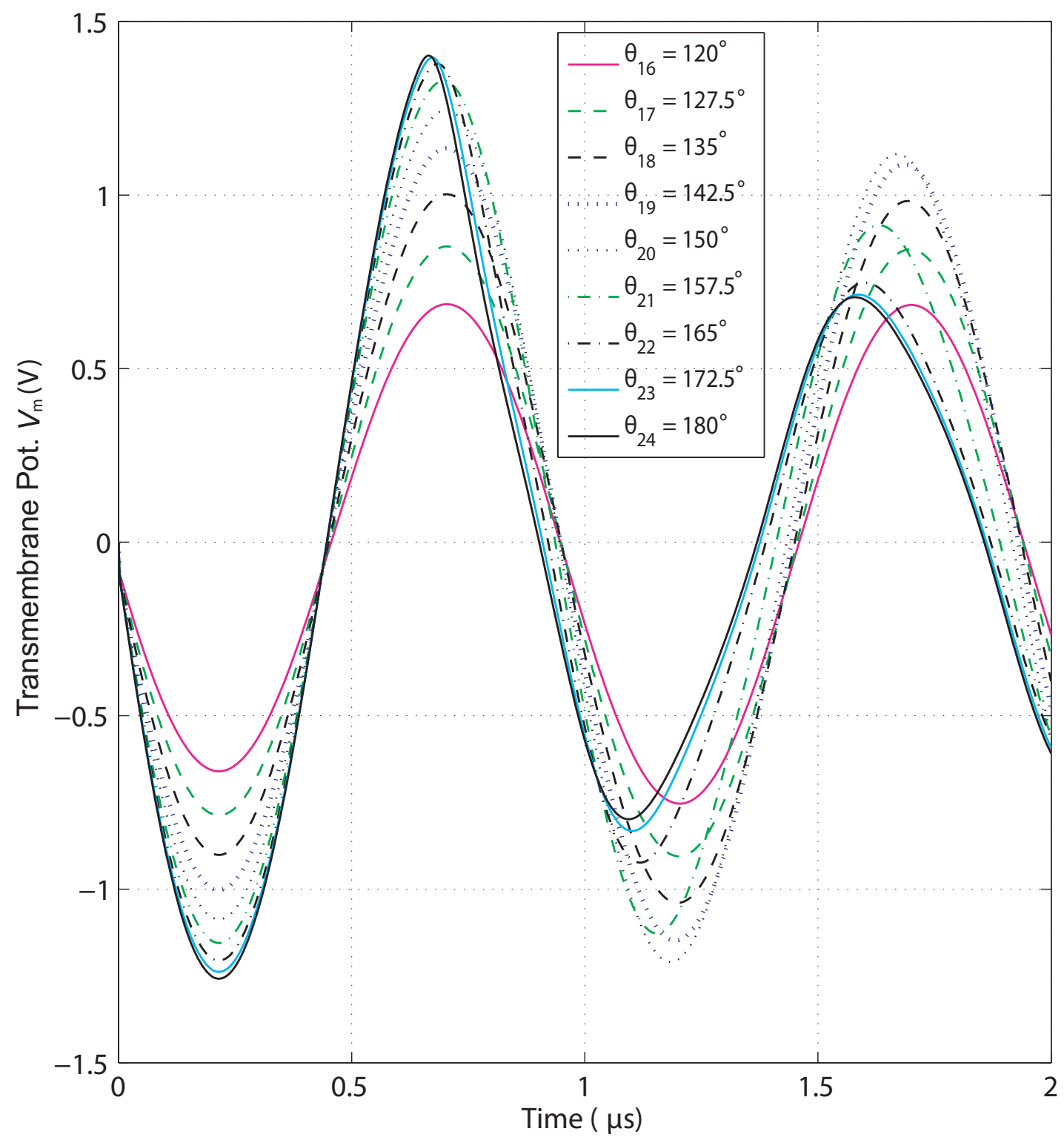

Figure 7: Transmembrane potential evolution at angles $120^{\circ}$ to $180^{\circ}$ around the cell membrane for a twocycle $1 \mathrm{MHz}$ sinusoidal bipolar applied electric field pulse with $235 \mathrm{kV} / \mathrm{m}$ peak amplitude. 

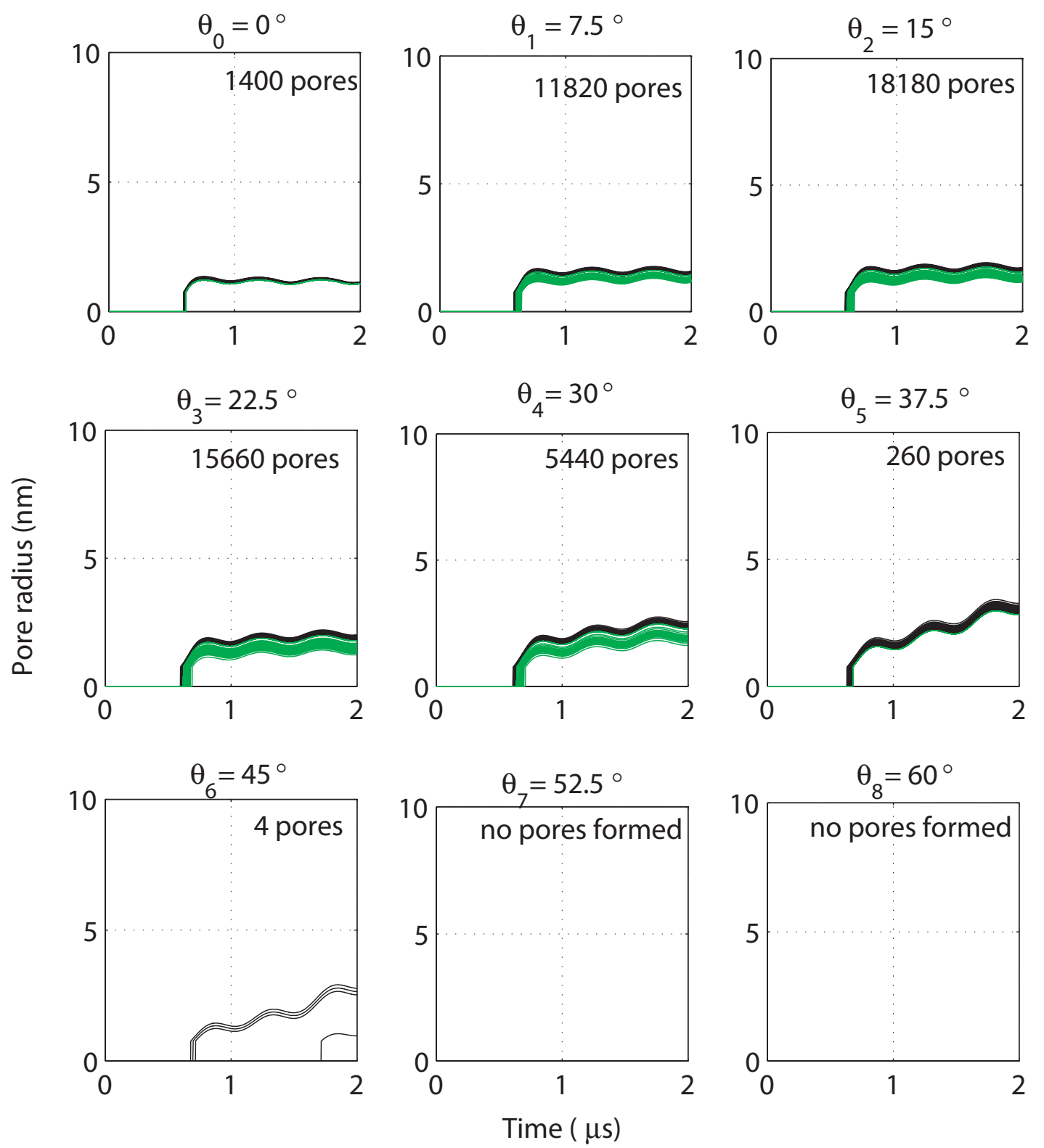

Figure 8: Pore radius evolution at angles $0^{\circ}$ to $60^{\circ}$ around the cell membrane for a two-cycle $1 \mathrm{MHz}$ sinusoidal bipolar applied electric field pulse with $235 \mathrm{kV} / \mathrm{m}$ peak amplitude. Black lines represent individual pores, green(grey) lines represent groups of 20 pores. 

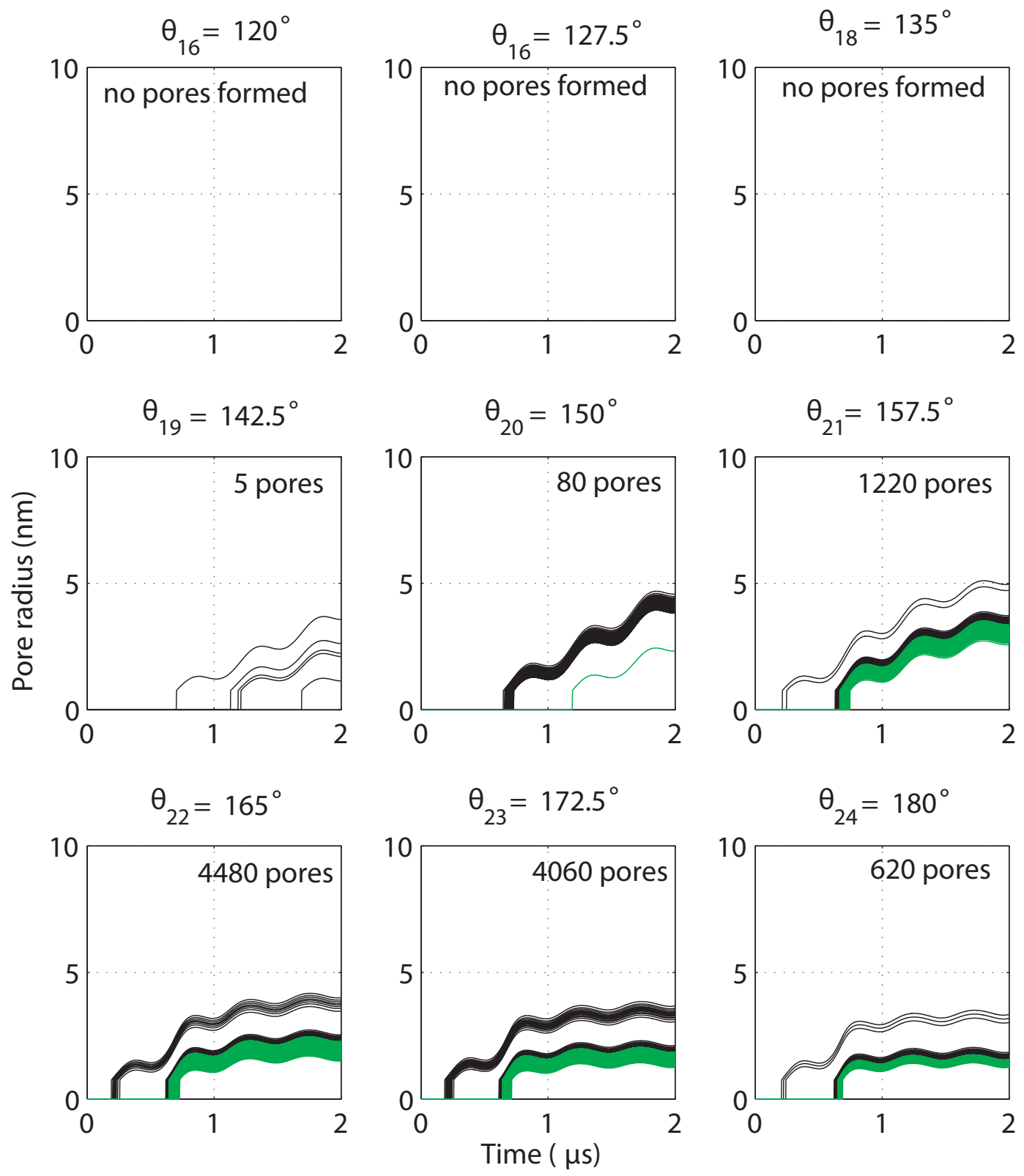

Figure 9: Pore radius evolution at polar angles $120^{\circ}$ to $180^{\circ}$ around the cell membrane for a two-cycle 1 $\mathrm{MHz}$ sinusoidal bipolar applied electric field pulse with $235 \mathrm{kV} / \mathrm{m}$ peak amplitude. Black lines represent individual pores, green(grey) lines represent groups of 20 pores. 


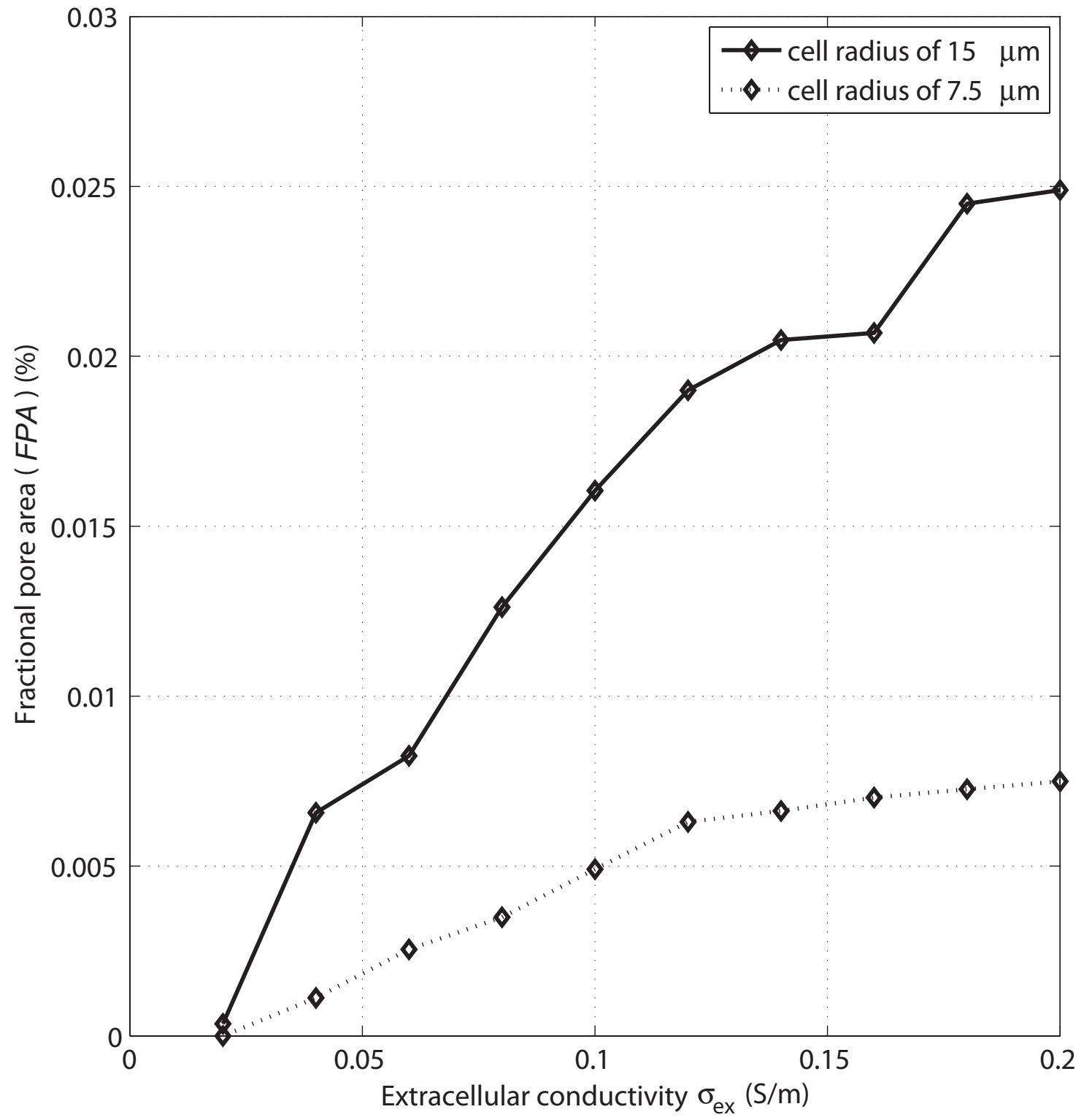

Figure 10: Fractional pore area versus extracellular conductivity for $7.5 \mu \mathrm{m}$, and $15 \mu \mathrm{m}$ cell radius exposed to a two-cycle sinusoidal bipolar applied electric field pulse of $130 \mathrm{kV} / \mathrm{m}$ peak magnitude at $100 \mathrm{kHz}$. 


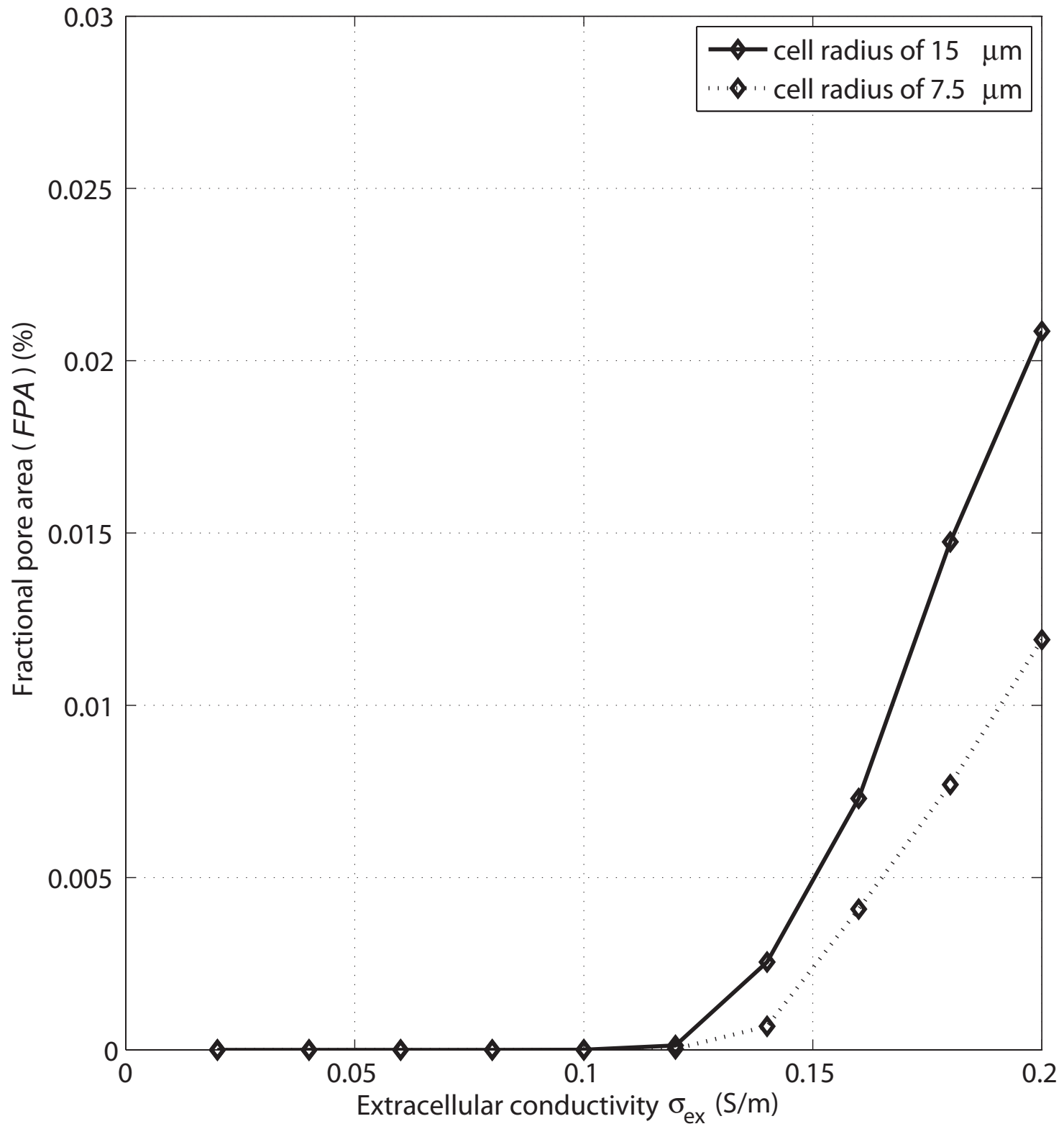

Figure 11: Fractional pore area versus extracellular conductivity for $7.5 \mu \mathrm{m}$, and $15 \mu \mathrm{m}$ cell radius exposed to a two-cycle sinusoidal bipolar applied electric field pulse of $350 \mathrm{kV} / \mathrm{m}$ peak magnitude at $1 \mathrm{MHz}$ 